MATHEMATICS OF COMPUTATION

Volume 68, Number 227, Pages 1201-1231

S 0025-5718(99)01051-0

Article electronically published on February 10, 1999

\title{
CALCULATION OF VALUES OF $L$-FUNCTIONS ASSOCIATED TO ELLIPTIC CURVES
}

\author{
SHIGEKI AKIYAMA AND YOSHIO TANIGAWA
}

\begin{abstract}
We calculated numerically the values of $L$-functions of four typical elliptic curves in the critical strip in the range $\operatorname{Im}(s) \leq 400$. We found that all the non-trivial zeros in this range lie on the critical line $\operatorname{Re}(s)=1$ and are simple except the one at $s=1$. The method we employed in this paper is the approximate functional equation with incomplete gamma functions in the coefficients. For incomplete gamma functions, we continued them holomorphically to the right half plane $\operatorname{Re}(s)>0$, which enables us to calculate for large $\operatorname{Im}(s)$. Furthermore we remark that a relation exists between Sato-Tate conjecture and the generalized Riemann Hypothesis.
\end{abstract}

\section{INTRODUCTION AND THE STATEMENT OF RESULTS}

The numerical calculations of the Riemann zeta function $\zeta(s)$ have a long history. In the critical strip, the Euler-Maclaurin summation formula is applicable, but on the critical line, the famous Riemann-Siegel formula is useful because it is very fast and accurate (see [3] or [8]). Using these formulas, it is known at present that the Riemann Hypothesis holds for $\operatorname{Im}(s)$ less than about $1.5 \times 10^{9}$ (see J. van de Lune, H. J. J. te Riele and D. T. Winter [13]; see also Odlyzko [16]). By the EulerMaclaurin summation formula, we can also calculate the values of the Hurwitz zeta function and hence the values of the Dirichlet $L$-function because it is a finite sum of the Hurwitz zeta functions.

For other $L$-functions, we have the examples of Manin [14], [15], Yoshida [24], [25] and Fermigier [4]. In his papers, Manin developed the theory of modular symbols and applied his theory to the calculation of Fourier coefficients of cusp forms of weight 2 and the value at 1 of the corresponding Dirichlet series. In fact, four examples of modular curves were treated in [15]. See Cremona [1] for other examples.

Yoshida and Fermigier calculated the values of the $L$-function on the critical line. Yoshida makes use of the iteration of partial summations in order to access the speed of convergence of the Dirichlet series. He said that his method "may seem to be only speculative" (p. 89 in [24]) and "on heuristic grounds" (p. 91 in [24]), though he made some discussions on the confidence of his data. He found zeros of many $L$-functions, namely, the $L$-function associated with the Ramanujan function $\Delta(z)$, the symmetric $j$-th power $L$-functions of $\Delta(z)$ for $j=2,3$ and 4 , $L$-functions associated to cusp forms, $L$-functions associated to Hecke characters of

Received by the editor May 22, 1996 and, in revised form, December 11, 1996.

1991 Mathematics Subject Classification. Primary 11F11, 11G40, 11M26.

Key words and phrases. Elliptic curve, $L$-function, approximate functional equation, Sato-Tate conjecture, Riemann Hypothesis. 
real quadratic fields and Artin's $L$-functions. He also observed that the generalized Riemann Hypothesis is true for $L(s, \Delta)$ in $0 \leq \operatorname{Im}(s) \leq 100$.

On the other hand, Fermigier used an expression of an $L$-function by the Dirichlet series with incomplete gamma functions in the coefficients. He employed the Romberg integration method for the calculation of incomplete gamma functions. He calculated the zeros of $L(1+i t, E)$ in $0 \leq t \leq 15$ for all the elliptic curves of prime conductor $N \leq 13100$. He also observed that the generalized Riemann Hypothesis is true for these $L(s, E)$ in $0 \leq \operatorname{Im}(s) \leq 15$.

The aim of this paper is to show a method and some examples for larger $\operatorname{Im}(s)$ than theirs. As in Fermigier [4], we use an expression of an $L$-function with the incomplete gamma function $\Gamma(s, z)$. However, the second variable $z$ is real in his case, so it seems somewhat difficult to calculate when $\operatorname{Im}(s)$ is large because the necessary increase in the memory bank of a machine is of exponential order with respect to $\operatorname{Im}(s)$. We continue the incomplete gamma function in the right half plane $\operatorname{Re}(s)>0$. After this, the increase is of polynomial order, hence we can calculate the value numerically for rather large $\operatorname{Im}(s)$. See the paragraphs after Theorem 1 below. After the analytic continuation, we expand it to the continued fraction. Using this method, we calculated the values of $L(1+i t, E)$ for four typical examples of elliptic curves in the range $0 \leq t \leq 400$, and checked that the generalized Riemann Hypothesis holds in this range.

We also present some graphs drawn by plotting the values of an $L$-function on the Gaussian plane. Since $L(s, E)$ has a functional equation, we can easily determine the argument of $L(s, E)$ on the critical line. So one may think that the essential thing is the absolute value, and such graph is meaningless. But the authors believe that such a visualization gives us the beauty and sense of the Riemann Hypothesis.

To state Theorem 1 , we shall recall the definition of the $L$-function associated to an elliptic curve. Let $E$ be an elliptic curve given by the global minimal Weierstrass equation

$$
y^{2}+A_{1} x y+A_{3} y=x^{3}+A_{2} x^{2}+A_{4} x+A_{6},
$$

and let $\Delta$ be its discriminant. For each prime $p$, we put

$$
a_{p}=p+1-\sharp E_{p}(\mathbf{Z} / p \mathbf{Z}),
$$

where $E_{p}$ is the reduction modulo $p$. If $p \mid \Delta$, then $E_{p}$ has a singularity and

$$
a_{p}=\left\{\begin{aligned}
0 & \text { for the case of a cusp } \\
1 & \text { for the case of a split node } \\
-1 & \text { for the case of a non-split node. }
\end{aligned}\right.
$$

If $p \nmid \Delta$, then we have

$$
\left|a_{p}\right| \leq 2 \sqrt{p}
$$

(Hasse's theorem). The $L$-function associated to $E$ is defined by

$$
L(s, E)=\prod_{p \mid \Delta} \frac{1}{1-a_{p} p^{-s}} \prod_{p \nmid \Delta} \frac{1}{1-a_{p} p^{-s}+p^{1-2 s}} .
$$

This infinite product is absolutely convergent for $\operatorname{Re} s>3 / 2$ by (1), and there we can expand it into the Dirichlet series $L(s, E)=\sum_{n=1}^{\infty} a_{n} n^{-s}$.

Assume that $E$ has a modular parametrization of level $N$. In the recent works of Wiles [23], Taylor and Wiles [19] and Diamond [2], it was proved that any elliptic 
curve which has semi-stable good reduction at 3 and 5 is modular. All the curves we treat in Section 5 satisfy this assumption. Let

$$
f_{E}(z)=\sum_{n=1}^{\infty} a_{n} e^{2 \pi i n z}
$$

then by Eichler-Shimura's theory, $f_{E}(z)$ is a primitive form of weight 2 with respect to $\Gamma_{0}(N)$. Furthermore we have

$$
f_{E}\left(-\frac{1}{N z}\right)=\mu N z^{2} f_{E}(z) \quad(\mu= \pm 1)
$$

Now, $L(s, E)$ can be continued holomorphically to the whole complex plane and satisfies the functional equation:

$$
N^{s / 2}(2 \pi)^{-s} \Gamma(s) L(s, E)=-\mu N^{(2-s) / 2}(2 \pi)^{-(2-s)} \Gamma(2-s) L(2-s, E) .
$$

The readers may refer to Knapp [10] for these subjects.

Then our first theorem is

Theorem 1. Let $s=\sigma+i$ be a complex number such that $1 / 2 \leq \sigma \leq 3 / 2$ and $t>0$ and let $\Gamma(s, z)$ be the incomplete gamma function of the second kind. We take a positive integer $M$ satisfying $M>t \sqrt{N} / 4$, and put $r=e^{i(\pi / 2-\delta(t))}$, where $\delta(t)$ is a function of $t$ with $0<\delta(t) \leq \pi / 2$. Then we have

$$
\begin{aligned}
L(s, E)= & \frac{1}{\Gamma(s)} \sum_{n \leq M} \frac{a_{n}}{n^{s}} \Gamma\left(s, \frac{2 \pi n r}{\sqrt{N}}\right) \\
& -\frac{\mu N^{1-s}(2 \pi)^{2(s-1)}}{\Gamma(s)} \sum_{n \leq M} \frac{a_{n}}{n^{2-s}} \Gamma\left(2-s, \frac{2 \pi n}{\sqrt{N} r}\right) \\
& +(2 \pi)^{s} \Gamma(s)^{-1} R,
\end{aligned}
$$

where the last term $R$ satisfies the inequality

$$
\begin{aligned}
& |R|<e^{-\pi t / 2} e^{\delta(t)(t-4 M / \sqrt{N})} N^{(1-\sigma) / 2} \sqrt{M} \delta(t)^{-1} \\
& \quad \times\left\{1+\frac{\log M+\sigma+1}{2 t \delta(t)}+\frac{(\sigma-1)(\log M+2)}{4(t \delta(t))^{2}}\right\} .
\end{aligned}
$$

The proof will be given in Section 2 .

The incomplete gamma function of the second kind is defined by

$$
\Gamma(s, z)=\int_{z}^{\infty} e^{-t} t^{s-1} d t \quad(z \in \mathbf{R}, \operatorname{Re}(s)>0) .
$$

(Note that this integral is denoted by $\Gamma(z, s)$ in Fermigier [4].) Following Lavrik [12], Karatsuba [9] and Turganaliev [21], we continue the function $\Gamma(s, z)$ holomorphically to the right half plane $\operatorname{Re}(z)>0$ by making a change of variable $t \rightarrow z t$ and rotating the line of integration by the $\operatorname{angle} \arg z$. Then we have

$$
\Gamma(s, z)=z^{s} \int_{1}^{\infty} e^{-z t} t^{s-1} d t
$$

and, when $\operatorname{Re}(s)$ is bounded and $\operatorname{Re}(z)>c$ for some positive constant $c$,

$$
|\Gamma(s, z)| \ll\left|z^{s}\right|
$$

uniformly in $s$ and $z$. 
The inequality (3) shows that when $\delta(t)$ is small, we must take large $M$ to get the accuracy of data. Hence, $\delta(t)=\pi / 2$ (this means $r=1$ ) is the most efficient choice for calculations theoretically. But, for a technical reason, we have to choose the function $\delta(t)<\pi / 2$. The function of the form $\Gamma(s, z) / \Gamma(s)$ appears in the right hand side of (2). Since $\Gamma(s)$ is of exponential decay when $|t| \rightarrow \infty$, the absolute value of each term in the sum may be very large. In fact, if $r=1$ and $t$ is large, each term in (2) is a huge number and it becomes impossible to compute it. In order to avoid this difficulty, we put $r=e^{i(\pi / 2-\delta(t))}$ and $\delta(t)<\pi / 2$. Then from $(6)$,

$$
|\Gamma(s, A r)| \ll A^{\sigma} e^{-\left(\frac{\pi}{2}-\delta(t)\right) t},
$$

(where $A$ is independent of $t$ ), hence the factor $e^{-\pi t / 2}$ cancels the one arising from the denominator. For example, if $\delta(t) \sim 1 / t, \Gamma(s, A r) / \Gamma(s)$ has a polynomial order as $|t| \rightarrow \infty$, hence we can put the calculations in practice. Turganaliev [21] put $\delta(t)=1 /(1+t)$. We choose $\delta(t)=\pi / 2$ for small $t, \delta(t)=1 /\left(1+\log ^{2} t\right)$ for a little larger $t$ and $\delta(t)=1 /(1+t)$ otherwise.

The estimation (3) does not make sense when $t$ is very close to 0 . But, in this case, we set $\delta(t)=\pi / 2$ and can get the explicit estimation from (16).

Our method is applicable to the Dirichlet series with an appropriate functional equation. Explicit estimation of the coefficients is needed to get the concrete upper bound of the error term. For example, we can treat the Dedekind zeta function or the Hecke $L$-function of a cusp form.

Now we shall state the next theorem. Let $E$ be an elliptic curve defined over $\mathbf{Q}$ without complex multiplication. Define $\theta_{p} \in(0, \pi)$ by $a_{p}=2 \sqrt{p} \cos \left(\theta_{p}\right)$. Let $p_{n}$ be the $n$-th prime number, and consider the real sequence $x_{n}=\theta_{p_{n}} / \pi, \quad(n=1,2, \ldots)$. Let $g$ be a real valued strictly increasing function on the interval $[0,1]$ with $g(0)=0$ and $g(1)=1$. Define the discrepancy with respect to the distribution function $g$ by

$$
D_{K}^{(g)}\left(x_{n}\right)=\sup _{0 \leq \alpha \leq 1}\left|\frac{A\left([0, \alpha),\left(x_{n}\right), K\right)}{K}-g(\alpha)\right|,
$$

where

$$
A\left([0, \alpha),\left(x_{n}\right), K\right)=\sharp\left\{x_{n} \in[0, \alpha) ; 1 \leq n \leq K\right\} .
$$

Put

$$
S T(x)=x-\frac{\sin (2 \pi x)}{2 \pi} .
$$

We call $D_{K}^{(S T)}$ the discrepancy with respect to the Sato-Tate measure. The SatoTate conjecture asserts that $\lim _{K \rightarrow \infty} D_{K}^{(S T)}\left(x_{n}\right)=0$, which is easily shown to be equivalent to

$$
\frac{\sharp\left\{\theta_{p} \in[\alpha, \beta) ; p \leq X\right\}}{\sharp\{p ; p \leq X\}} \rightarrow \frac{2}{\pi} \int_{\alpha}^{\beta} \sin ^{2}(t) d t,
$$

for any $0 \leq \alpha \leq \beta \leq \pi$. See Ogg [17] or Shahidi [18] for the Sato-Tate conjecture. Here we propose a quantitative version of their conjecture.

Conjecture 1. For any positive $\varepsilon, D_{K}^{(S T)}\left(x_{n}\right)=O\left(K^{-1 / 2+\varepsilon}\right)$.

Then our second theorem is 
Theorem 2. Let $E$ be an elliptic curve defined over $\mathbf{Q}$ which has no complex multiplication. Then the above Conjecture implies the truth of the generalized Riemann Hypothesis for $L(s, E)$.

The proof of Theorem 2 will be given in Section 3 .

In Section 5, we show numerical experiments on Conjecture 1 for some elliptic curves. These seem to support the validity of Conjecture 1 .

\section{Approximate functional equation}

Let $f(z)$ be a cusp form of weight $k$ with respect to $\Gamma_{0}(N)$, and let $f(z)=$ $\sum_{n=1}^{\infty} a_{n} e^{2 \pi i n z}$ be the Fourier expansion at the cusp $\infty$. Furthermore we assume that $f(z)$ is an eigenfunction of the involution

$$
\left.f \rightarrow f\right|_{k}\left(\begin{array}{cc}
0 & -1 \\
N & 0
\end{array}\right)
$$

with eigenvalue $\mu= \pm 1$; namely,

$$
f\left(-\frac{1}{N z}\right)=\mu N^{k / 2} z^{k} f(z) .
$$

Let $L(s, f)$ be the Dirichlet series defined by $L(s, f)=\sum_{n=1}^{\infty} a_{n} n^{-s}$. Since $a_{n}=$ $O\left(n^{\frac{k-1}{2}+\varepsilon}\right)$ by Deligne's theorem, $L(s, f)$ converges absolutely for $\operatorname{Re} s>(k+1) / 2$. It is well known that the function $\Lambda(s, f):=N^{s / 2}(2 \pi)^{-s} \Gamma(s) L(s, f)$ is an entire function of $s$ and satisfies the functional equation

$$
\Lambda(s, f)=\mu i^{k} \Lambda(k-s, f) .
$$

By modifying the standard proof of (9), we get the approximate functional equation with incomplete gamma functions. Let $I(s)$ be the function defined by

$$
I(s)=\int_{0}^{\infty} y^{s-1} f(i y) d y
$$

which is equal to $(2 \pi)^{-s} \Gamma(s) L(s, f)$ for $\operatorname{Re} s>(k+1) / 2$. Let $r$ be a complex number with $\operatorname{Re}(r)>0$, and divide the integral of $I(s)$ as

$$
I(s)=\int_{0}^{\infty} y^{s-1} f(i y) d y=\int_{0}^{\frac{r}{\sqrt{N}}}+\int_{\frac{r}{\sqrt{N}}}^{\infty} .
$$

Then

$$
\int_{\frac{r}{\sqrt{N}}}^{\infty} y^{s-1} f(i y) d y=N^{-s / 2} r^{s} \sum_{n=1}^{\infty} a_{n} \int_{1}^{\infty} y^{s-1} e^{-\frac{2 \pi n r y}{\sqrt{N}}} d y .
$$

On the other hand, the equation (8) gives us

$$
\begin{aligned}
\int_{0}^{\frac{r}{\sqrt{N}}} y^{s-1} f(i y) d y & =N^{-s} \int_{\frac{1}{\sqrt{N r}}}^{\infty} y^{-s-1} f\left(\frac{i}{N y}\right) d y \\
& =\mu i^{k} N^{-s / 2} r^{s-k} \sum_{n=1}^{\infty} a_{n} \int_{1}^{\infty} y^{k-s-1} e^{-\frac{2 \pi n y}{\sqrt{N r}}} d y
\end{aligned}
$$


This shows the analytic continuation of $I(s)$ to the whole complex plane. The last integrals in (10) and (11) can be written as

$$
\left(\frac{2 \pi n r}{\sqrt{N}}\right)^{-s} \Gamma\left(s, \frac{2 \pi n r}{\sqrt{N}}\right) \text { and }\left(\frac{\sqrt{N} r}{2 \pi n}\right)^{k-s} \Gamma\left(k-s, \frac{2 \pi n}{\sqrt{N} r}\right),
$$

respectively. Hence we obtain the following

Lemma 1. Let $M$ be an arbitrary positive integer, then we have

$$
\begin{aligned}
L(s, f)= & \frac{1}{\Gamma(s)} \sum_{n \leq M} \frac{a_{n}}{n^{s}} \Gamma\left(s, \frac{2 \pi n r}{\sqrt{N}}\right) \\
& +\frac{\mu i^{k} N^{k / 2-s}(2 \pi)^{2 s-k}}{\Gamma(s)} \sum_{n \leq M} \frac{a_{n}}{n^{k-s}} \Gamma\left(k-s, \frac{2 \pi n}{\sqrt{N} r}\right) \\
& +(2 \pi)^{s} \Gamma(s)^{-1} R,
\end{aligned}
$$

where

$$
\begin{aligned}
R=N^{-s / 2} r^{s} \sum_{n>M} a_{n} \int_{1}^{\infty} y^{s-1} e^{-\frac{2 \pi n r y}{\sqrt{N}}} d y \\
\quad+\mu i^{k} N^{-s / 2} r^{s-k} \sum_{n>M} a_{n} \int_{1}^{\infty} y^{k-1-s} e^{-\frac{2 \pi n y}{\sqrt{N r}}} d y
\end{aligned}
$$

Now let us consider the case $k=2$. We assume that $f(z)$ is a primitive form. The error term $R$ in (12) can be written as

$$
\begin{aligned}
R= & N^{-s / 2} r^{s} \sum_{n>M} a_{n} \int_{1}^{\infty} y^{s-1} e^{-\frac{2 \pi n r y}{\sqrt{N}}} d y \\
& -\mu N^{-s / 2} r^{s-2} \sum_{n>M} a_{n} \int_{1}^{\infty} y^{1-s} e^{-\frac{2 \pi n y}{\sqrt{N}}} d y .
\end{aligned}
$$

As is stated in Section 1, we put

$$
r=e^{i(\pi / 2-\delta(t))},
$$

where $\delta(t)$ is a function of $t$ satisfying $0<\delta(t) \leq \pi / 2$. Then

$$
\begin{aligned}
|R| \leq N^{-\sigma / 2} e^{-(\pi / 2-\delta(t)) t}\left\{\sum_{n>M}\left|a_{n}\right|\right. & \int_{1}^{\infty} y^{\sigma-1} e^{-\frac{2 \pi n \sin \delta(t)}{\sqrt{N}} y} d y \\
& \left.+\sum_{n>M}\left|a_{n}\right| \int_{1}^{\infty} y^{1-\sigma} e^{-\frac{2 \pi n \sin \delta(t)}{\sqrt{N}} y} d y\right\} .
\end{aligned}
$$

We may assume that $1 \leq \sigma<3 / 2$, and we write $\lambda=2 \pi \sin \delta(t)$ for simplicity. The integrals in the right hand side of the above inequality are evaluated as

$$
\int_{1}^{\infty} y^{\sigma-1} e^{-\frac{\lambda n y}{\sqrt{N}}} d y \leq \frac{\sqrt{N}}{\lambda n} e^{-\frac{\lambda n}{\sqrt{N}}}+\frac{N(\sigma-1)}{(\lambda n)^{2}} e^{-\frac{\lambda n}{\sqrt{N}}}
$$

and

$$
\int_{1}^{\infty} y^{1-\sigma} e^{-\frac{\lambda n y}{\sqrt{N}}} d y \leq \frac{\sqrt{N}}{\lambda n} e^{-\frac{\lambda n}{\sqrt{N}}}
$$


We also have $\left|a_{n}\right| \leq \sqrt{n} d(n)$. Hence we get

$$
\begin{aligned}
|R| \leq & 2 N^{(1-\sigma) / 2} e^{-(\pi / 2-\delta(t)) t} \lambda^{-1} \sum_{n>M} \frac{d(n)}{n^{1 / 2}} e^{-\frac{\lambda n}{\sqrt{N}}} \\
& +(\sigma-1) N^{1-\sigma / 2} e^{-(\pi / 2-\delta(t)) t} \lambda^{-2} \sum_{n>M} \frac{d(n)}{n^{3 / 2}} e^{-\frac{\lambda n}{\sqrt{N}}} .
\end{aligned}
$$

To evaluate the summations in the right hand side, we put $D(x)=\sum_{n \leq x} d(n)$. By the elementary fact

$$
x(\log x-1) \leq D(x) \leq x(\log x+1)
$$

and partial summation, we get

$$
\begin{aligned}
& \sum_{n>M} \frac{d(n)}{n^{1 / 2}} e^{-\frac{\lambda n}{\sqrt{N}}}=-D(M) M^{-1 / 2} e^{-\frac{\lambda M}{\sqrt{N}}}-\int_{M}^{\infty} D(u) \frac{d}{d u}\left(u^{-1 / 2} e^{-\frac{\lambda u}{\sqrt{N}}}\right) d u \\
& \leq-\sqrt{M}(\log M-1) e^{-\frac{\lambda M}{\sqrt{N}}}+\int_{M}^{\infty}(\log u+1)\left(\frac{\lambda}{\sqrt{N}} \sqrt{u}+\frac{1}{2 \sqrt{u}}\right) e^{-\frac{\lambda u}{\sqrt{N}}} d u \\
& \quad \leq\left(\frac{\log M+2}{\lambda} \sqrt{\frac{N}{M}}+2 \sqrt{M}\right) e^{-\frac{\lambda M}{\sqrt{N}}} .
\end{aligned}
$$

Similarly, we have that

$$
\sum_{n>M} \frac{d(n)}{n^{3 / 2}} e^{-\frac{\lambda n}{\sqrt{N}}} \leq\left(\frac{2}{\sqrt{M}}+\frac{\log M+2}{\lambda M} \sqrt{\frac{N}{M}}\right) e^{-\frac{\lambda M}{\sqrt{N}}}
$$

From (13), (14) and (15), we get

$$
\begin{array}{r}
|R| \leq e^{-\frac{\pi}{2} t} e^{\left(t \delta(t)-\frac{\lambda M}{\sqrt{N}}\right)} N^{(1-\sigma) / 2}\left\{\frac{4 \sqrt{M}}{\lambda}+\frac{2(\log M+\sigma+1)}{\lambda^{2}} \sqrt{\frac{N}{M}}\right. \\
\left.+\frac{(\sigma-1)(\log M+2)}{\lambda^{3} \sqrt{M}} \frac{N}{M}\right\} .
\end{array}
$$

Now take $M \geq t \sqrt{N} / 4$ and use the inequality $\lambda>4 \delta(t)$, then we get Theorem 1 .

\section{Proof of Theorem 2}

To prove Theorem 2, we need the following lemma of Koksma.

Lemma 2 (Koksma's inequality). Let $f$ be a real valued function on $[0,1]$. Suppose that $f$ has bounded variation. Let $g$ be a real valued continuous strictly increasing function on $[0,1]$ with $g(0)=0$ and $g(1)=1$. Then we have

$$
\left|\frac{1}{K} \sum_{n=1}^{K} f\left(x_{n}\right)-\int_{0}^{1} f(t) d g(t)\right| \leq D_{K}^{(g)}\left(x_{n}\right) V(f),
$$

for any sequence of real numbers $\left(x_{n}\right)_{n=1,2, \ldots}$ in $[0,1]$. Here $V(f)$ is the total variation of $f$ in $[0,1]$ and $D_{K}^{(g)}\left(x_{n}\right)$ is the discrepancy defined by $(7)$.

Proof. See Kuipers and Niederreiter [11, p. 142] for the case $g(t)=t$. It is an easy exercise to generalize it to our case. 
Now we prove Theorem 2. Put

$$
A(s)=\prod_{p \nmid \Delta} \frac{1}{1-a_{p} p^{-s}+p^{1-2 s}}
$$

for $\operatorname{Re} s>3 / 2$. We note that

$$
\log A(s)=\sum_{p} a_{p} p^{-s}+O\left(\sum_{p} p^{1-2 \sigma}\right) .
$$

Since the error term is holomorphic in $\operatorname{Re} s>1 / 2$, we have only to consider the $\operatorname{sum} \sum_{p} a_{p} p^{-s}=2 \sum_{p} \cos \left(\theta_{p}\right) p^{1 / 2-s}$. (Here we can neglect the bad primes.) If this sum is holomorphic in $\operatorname{Re} s>1$, then $L(s, E)$ has no zeros in $\operatorname{Re} s>1$, which is the generalized Riemann Hypothesis for $L(s, E)$. By partial summation, we see that if

$$
\sum_{p<x} \cos \left(\theta_{p}\right)=O\left(x^{1 / 2+\varepsilon}\right) \quad \text { for any } \quad \varepsilon>0,
$$

then $\log A(s)$ is holomorphic in $\operatorname{Re} s>1$. Now, let $f(t)=\cos (\pi t)$ and $g(t)=S T(t)$ in Lemma 2. Then we have

$$
\left|\frac{1}{K} \sum_{n=1}^{K} \cos \left(\theta_{p_{n}}\right)\right| \leq 2 D_{K}^{(S T)}\left(x_{n}\right) .
$$

Hence, Conjecture 1 implies that $\sum_{n=1}^{K} \cos \left(\theta_{p_{n}}\right)=O\left(K^{1 / 2+\varepsilon}\right)$. Combining this and (18), we see that (17) holds, and we get the desired result.

\section{Continued fraction of the incomplete gamma function}

The problem is that the calculation of incomplete gamma functions is very difficult and needs much time. Here it is appropriate to explain how to evaluate it with satisfactory accuracy. Let $\Gamma(s, z)$ be the incomplete gamma function of the second kind defined by (4), and let

$$
\gamma(s, z)=\int_{0}^{z} e^{-t} t^{s-1} d t=\Gamma(s)-\Gamma(s, z)
$$

be the incomplete gamma function of the first kind.

When $z$ has a small absolute value, we can use the Taylor expansion:

$$
\gamma(s, z)=\sum_{n=0}^{\infty} \frac{(-1)^{n} z^{s+n}}{n !(s+n)},
$$

or the formula due to Legendre:

$$
\gamma(s, z)=e^{-z} \sum_{n=0}^{\infty} \frac{\Gamma(s) z^{s+n}}{\Gamma(s+n+1)} .
$$

When $|z|$ is large enough, we use the formula

$$
\Gamma(s, z)=z^{s-1} e^{-z}\left(1+\sum_{n=1}^{\infty} \frac{1}{z^{n}}(s-1)(s-2) \cdots(s-n)\right) .
$$


Note that the series of the right hand side is divergent and this equality should be considered as the asymptotic expansion at $z=\infty$. To calculate approximate values of $\gamma(s, z)$ and $\Gamma(s, z)$, we employ their classical continued fraction expansions:

$$
\gamma(s, z)=z^{s} e^{-z} s-\frac{1}{s+1+\frac{1 \cdot z}{s+2-\frac{(s+1) z}{s+3+\frac{2 \cdot z}{s+4-\frac{(s+2) z}{s+5+\frac{3 \cdot z}{s+6-} \cdot}}}}}
$$

and

$$
\Gamma(s, z)=z^{s} e^{-z} \frac{1}{z+\frac{1-s}{1+\frac{1}{z+\frac{2-s}{1+\frac{2}{z+\frac{3-s}{1+\frac{3}{z+} \cdot}}}}}}
$$

These formulas are special cases of Gauss's continued fraction method using confluent hypergeometric functions (see Jones and Thron [5], pp. 205-209 and pp. 344-348 or Wall [22]). As a formal power series of $z$ and $s$, (22) (resp. (23)) is equivalent to (20) (resp. (21)). However, the continued fraction in (22) is convergent for any $z$ and $s$ with $\operatorname{Re} s>0$, and the one in (23) for $z$ with $|\arg z|<\pi$ and $s \neq 1,2,3, \ldots$.

To estimate the truncation error of these continued fraction expansions, we quote the result of [5]. Let $\theta, \xi_{-1}$ be real numbers with $0<|\theta|<\pi$ and let $\left\{a_{n}\right\}_{n=0,1,2, \ldots}$ be a sequence of arbitrary non-zero complex numbers. We define $\xi_{n}=\arg a_{n}-\xi_{n-1}-\theta$ recursively. Let $\left\{b_{n}\right\}_{n=0,1,2, \ldots}$ be another sequence of complex numbers satisfying the conditions

$$
\begin{aligned}
& 0 \leq \arg b_{n}-\xi_{n} \leq \theta \quad \text { if } \quad 0<\theta<\pi, \\
& \theta \leq \arg b_{n}-\xi_{n} \leq 0 \quad \text { if } \quad-\pi<\theta<0 .
\end{aligned}
$$

Consider the continued fraction

$$
\frac{a_{0}}{b_{0}+\frac{a_{1}}{b_{1}+\frac{a_{2}}{b_{2}+\ddots}}}
$$

Then Jones and Thron (see Th. 8.8 in [5] and [6]) proved the following 
Theorem 3 (Jones and Thron). If the continued fraction (24) converges to a value $x$, then

$$
\begin{array}{ll}
\left|x-\frac{P_{n}}{Q_{n}}\right| \leq\left|\frac{P_{n}}{Q_{n}}-\frac{P_{n-1}}{Q_{n-1}}\right|, \quad \text { if } \quad 0<|\theta| \leq \frac{\pi}{2}, \\
\left|x-\frac{P_{n}}{Q_{n}}\right| \leq \frac{1}{|\sin \theta|}\left|\frac{P_{n}}{Q_{n}}-\frac{P_{n-1}}{Q_{n-1}}\right|, \quad \text { if } \quad \frac{\pi}{2}<|\theta|<\pi,
\end{array}
$$

where $P_{n} / Q_{n}(n=0,1,2, \ldots)$ is the $n$-th convergent of the continued fraction (24).

First we apply Theorem 3 to $(22)$. Let $P_{n} / Q_{n}(n=0,1,2, \ldots)$ be the $n$-th convergent of the continued fraction expansion of $\gamma(s, z) z^{-s} e^{z}$. Put

$$
b_{n}=\left\{\begin{array}{lll}
n z, & n & \text { even } \\
-(s+(n-1) / 2) z, & n & \text { odd }
\end{array}\right.
$$

Then it is easily seen that

$$
Q_{n}=(s+n+1) Q_{n-1}+b_{n} Q_{n-2},
$$

and

$$
P_{n} Q_{n-1}-P_{n-1} Q_{n}=-b_{n}\left(P_{n-1} Q_{n-2}-P_{n-2} Q_{n-1}\right)
$$

Thus we have

$$
\begin{aligned}
Q_{2 n-1} & =\sum_{k=0}^{n}(-1)^{k}\left(\begin{array}{l}
n \\
k
\end{array}\right) \frac{\Gamma(2 n-k+s)}{\Gamma(s)} z^{k} \\
Q_{2 n} & =\sum_{k=0}^{n}(-1)^{k}\left(\begin{array}{l}
n \\
k
\end{array}\right) \frac{\Gamma(2 n+1-k+s)}{\Gamma(s)} z^{k},
\end{aligned}
$$

and

$$
\frac{P_{2 n}}{Q_{2 n}}-\frac{P_{2 n-1}}{Q_{2 n-1}}=\frac{(-1)^{n} n ! \Gamma(s+n)}{\Gamma(s) Q_{2 n} Q_{2 n-1}}=\frac{(-1)^{n} n ! \Gamma(s)}{\Gamma(s+n) Q_{2 n}^{*} Q_{2 n-1}^{*}}
$$

where, in the right hand side of (25), we put

$$
\begin{aligned}
Q_{2 n-1}^{*} & =\sum_{k=0}^{n}(-1)^{k}\left(\begin{array}{l}
n \\
k
\end{array}\right) \frac{\Gamma(s+n+k)}{\Gamma(s+n)} z^{-k}, \\
Q_{2 n}^{*} & =\sum_{k=0}^{n}(-1)^{k}\left(\begin{array}{l}
n \\
k
\end{array}\right) \frac{\Gamma(s+n+1+k)}{\Gamma(s+n)} z^{-k},
\end{aligned}
$$

or in another way

$$
\begin{aligned}
Q_{2 n-1}^{*} & =\sum_{k=0}^{n}\left(\begin{array}{l}
n \\
k
\end{array}\right)\left(1+\frac{n+k-1}{s}\right)\left(1+\frac{n+k-2}{s}\right) \cdots\left(1+\frac{n}{s}\right)\left(-\frac{z}{s}\right)^{-k}, \\
Q_{2 n}^{*} & =\sum_{k=0}^{n}\left(\begin{array}{l}
n \\
k
\end{array}\right)\left(1+\frac{n+k}{s}\right)\left(1+\frac{n+k-1}{s}\right) \cdots\left(1+\frac{n}{s}\right)\left(-\frac{x}{s}\right)^{-k} .
\end{aligned}
$$


The error estimate of $(23)$ proceeds similarly. Let $U_{n} / V_{n}(n=0,1,2, \ldots)$ be the $n$-th convergent of $\Gamma(s, z) z^{-s} e^{z}$, then we can show by induction

$$
\begin{aligned}
V_{2 n-1} & =\sum_{k=0}^{n}\left(\begin{array}{l}
n \\
k
\end{array}\right) \frac{\Gamma(n+1-s)}{\Gamma(k+1-s)} x^{k}, \\
V_{2 n} & =\sum_{k=0}^{n}\left(\begin{array}{l}
n \\
k
\end{array}\right) \frac{\Gamma(n+1-s)}{\Gamma(k+2-s)} x^{k+1} .
\end{aligned}
$$

Thus we have

$$
\frac{U_{2 n}}{V_{2 n}}-\frac{U_{2 n-1}}{V_{2 n-1}}=\frac{n ! \Gamma(n+1-s)}{\Gamma(1-s) V_{2 n} V_{2 n-1}}=\frac{n ! \Gamma(1-s)}{\Gamma(n+2-s) V_{2 n}^{*} V_{2 n-1}^{*}},
$$

where

$$
\begin{aligned}
V_{2 n-1}^{*} & =\sum_{k=0}^{n}\left(\begin{array}{l}
n \\
k
\end{array}\right) \frac{\Gamma(1-s)}{\Gamma(k+1-s)} z^{k}, \\
V_{2 n}^{*} & =\sum_{k=0}^{n}\left(\begin{array}{l}
n \\
k
\end{array}\right) \frac{\Gamma(1-s)}{\Gamma(k+2-s)} z^{k+1},
\end{aligned}
$$

or similarly,

$$
\begin{aligned}
V_{2 n-1}^{*} & =\sum_{k=0}^{n}\left(\begin{array}{l}
n \\
k
\end{array}\right) \frac{(-z / s)^{k}}{\left(1-\frac{k}{s}\right)\left(1-\frac{k-1}{s}\right) \cdots\left(1-\frac{1}{s}\right)}, \\
V_{2 n}^{*} & =\sum_{k=0}^{n}\left(\begin{array}{l}
n \\
k
\end{array}\right) \frac{(-z / s)^{k+1}}{\left(1-\frac{k+1}{s}\right)\left(1-\frac{k}{s}\right) \cdots\left(1-\frac{1}{s}\right)} .
\end{aligned}
$$

Applying the Theorem of Jones and Thron, we can estimate the truncation error by (25) or (27). The expressions (26) and (28) suggest that there exists a kind of duality. Thus, to calculate the precise value of $\Gamma(s, z)$, it is better to use (23) when $|z| \geq|s|$ and (22) when $|z|<|s|$. Numerical experiments suggest that this choice is very suitable. Note that, if necessary, we must apply Theorem 3 to the sub-expression of (22) and (23) because the first finite terms may not satisfy the condition of Theorem 3.

\section{EXAMPLES}

In this section, we give examples of our calculations. In the graphs of zeta functions or $L$-functions below, the horizontal axis (resp. vertical axis) represents the real part (resp. imaginary part) of their values.

As a model, we first show two graphs of the Riemann zeta function on the critical line (Figures 1 and 2). The range of $t$ is written under each graph. Figures 3, 4, 5, and 6 are the graphs of the Hurwitz zeta function $\zeta(1 / 2+i t, j / 5),(j=1, \cdots, 4)$ for $50.00 \leq t \leq 60.00$. Figure 7 is the Dirichlet $L$-function $L(1 / 2+i t, \chi)$, where $\chi$ is the Dirichlet character mod 5 determined by $\chi(2)=\exp \left(2 \pi i \frac{3}{4}\right)$. It is interesting that the Hurwitz zeta function moves rather randomly but their sum, the Dirichlet $L$-function, moves as the Riemann zeta function. We calculated their values by the Euler-Maclaurin summation formula. 
For $L$-functions associated to elliptic curves, we study four examples with different Mordell-Weil ranks. Let

$$
\begin{aligned}
& E_{11}: y^{2}+y=x^{3}-x^{2}-10 x-20, \quad N=11, \quad r=0, \\
& E_{37}: y^{2}+y=x^{3}-x, \quad N=37, \quad r=1, \\
& E_{446}: y^{2}+x y=x^{3}-x^{2}-4 x+4, \quad N=446, \quad r=2, \\
& E_{5077}: y^{2}+y=x^{3}-7 x+6, \quad N=5077, \quad r=3,
\end{aligned}
$$

where $N$ and $r$ represent the conductor and the Mordell-Weil rank of $E$, respectively. (See Figures 8, 9, 10, 11, 13, 14, 16, 18, 17 and 19.) In the following subsections, we show the graphs of $L\left(s, E_{N}\right)$ and some other data. For these, we used the method of Sections 2 and 4.

Figures 12,15 and 20 are the graphs of $L\left(1+i t, E_{N}\right)$ when $t$ is close to 0 . By Tables 1,2 , and 3 , up to the numerical precision of the calculation, we can see that $L\left(s, E_{37}\right), L\left(s, E_{446}\right)$ and $L\left(s, E_{5077}\right)$ have zeros of order 1,2 and 3 at $s=1$, respectively. This observation is compatible with the Birch and Swinnerton-Dyer conjecture.

One can ask whether all non-trivial zeros lie on the critical line $\operatorname{Re}(s)=1$ or not. The generalized Riemann hypothesis insists that this is the case. As is stated in Section 1, Yoshida and Fermigier showed that it holds true in the range of their calculation. In our cases, we have

Theorem 4. The generalized Riemann Hypothesis is true for the above four elliptic curves in the range $\operatorname{Im}(s) \leq 400$. Moreover, all non-trivial zeros except the one at $s=1$ are simple.

Theorem 4 can be shown by the classical method of Backlund (cf. Edwards [3], Yoshida [24]). The zeros in this range are listed in Tables 4, 5 and 6.

Figures 21, 22 and 23 are the graphs of $L\left(\sigma+i t, E_{5077}\right) \quad 17.7 \leq t \leq 20.5$ for $\sigma=0.98,1.00,1.02$, respectively. It is interesting to see how $L(s, E)$ takes the value 0 on the critical line $\operatorname{Re}(s)=1$.

Figures 24, 25, 26 and 27 are numerical experiments of Conjecture 1 in Section 1. In these figures, the horizontal axis and vertical axis represent $K$ and $\sqrt{K} D_{K}^{(S T)}\left(x_{n}\right)$, respectively.

We used Kida's UBASIC86 on 80486-66Mhz first and Pentium-133Mhz later. We also used PARI for the calculations of $a_{n}$ and Mathematica for drawing the graphs. According to Theorem 1, time evaluation for the calculation of $L\left(1+i t, E_{N}\right)$ is $O(t \sqrt{N})$ if we ignore the incomplete gamma function. But the bottleneck is the calculation of the incomplete gamma function. It takes an enormous amount of time at the present stage. For example, on $80486-66 \mathrm{Mhz}$, it takes about 150 hours for $L\left(1+i t, E_{11}\right)$ and 900 hours for $L\left(1+i t, E_{5077}\right)$ with sufficiently many division points in the range $0 \leq t \leq 400$. So we did not aim to make a complete list here, leaving it as a future problem. But for a single $t$, even if it is large, we can calculate the value of $L(s, E)$ in the critical strip. For example,

$$
\begin{aligned}
& L\left(1+1000 i, E_{5077}\right)=0.97714-0.79882 i \\
& L\left(1+3000 i, E_{5077}\right)=1.87754-1.90091 i
\end{aligned}
$$


The correctness of our calculation is checked by comparison with Yoshida's data for $L\left(s, E_{11}\right)$. Moreover, for the above four curves, we checked the invariance of the data with $\delta(t)=1 /(1+t)$ and $\delta(t)=1 /\left(1+\log ^{2} t\right)$. (It seems to be a convenient way to check the correctness.)

One can find our programs on ftp://ftp.math.metro-u.ac.jp/tnt.

5.1. The Riemann zeta function: $\zeta(1 / 2+i t)$.

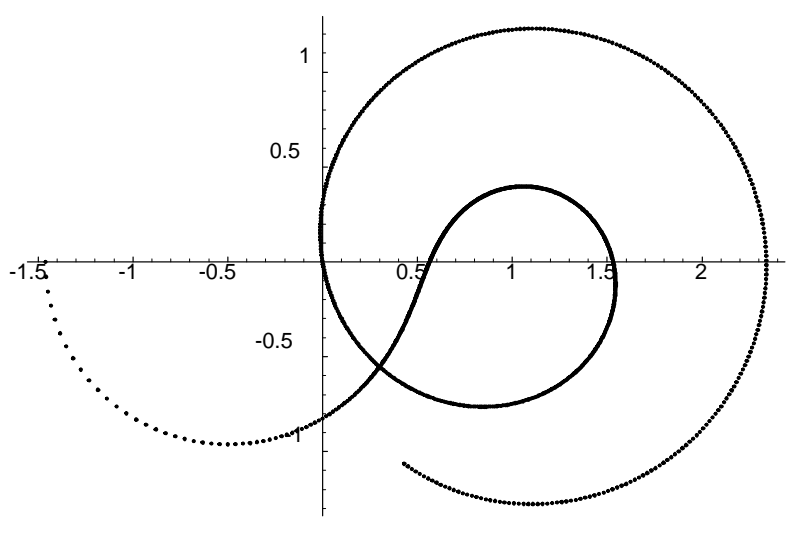

FiguRE $1 . \quad 0 \leq t \leq 20$

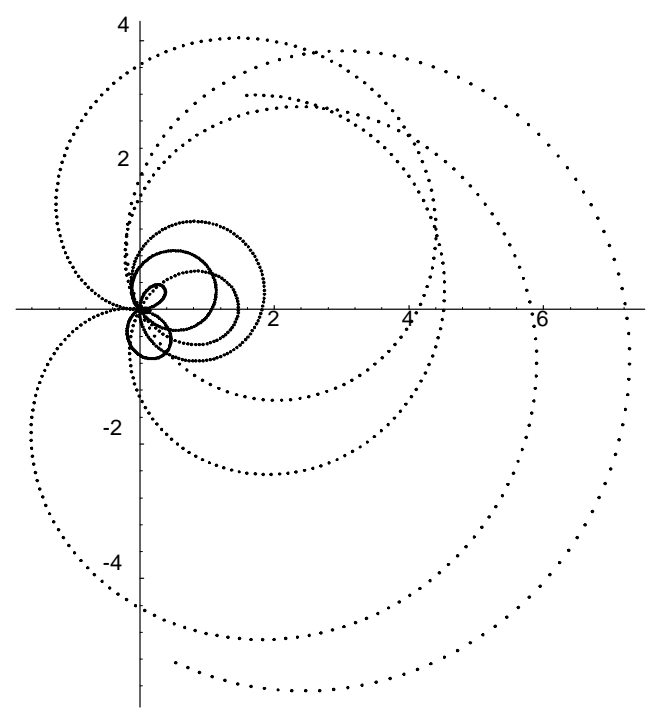

FIGURE 2. $\quad 3000 \leq t \leq 3010$

(The horizontal and vertical axes indicate the real and imaginary parts of the Riemann zeta function.) 
The Hurwitz zeta function: $\zeta(1 / 2+i t, \alpha)$.

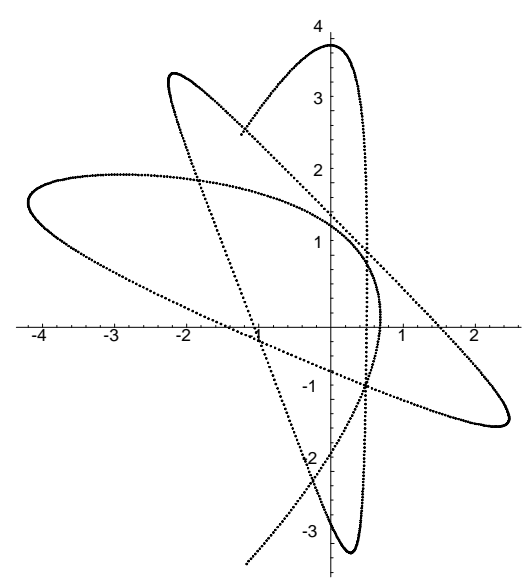

Figure 3. $\alpha=1 / 5$ for $50 \leq t \leq 60$

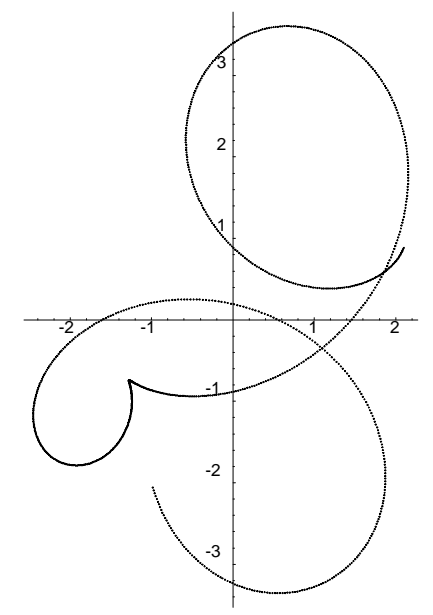

FiguRE 5. $\alpha=3 / 5$ for $50 \leq t \leq 60$

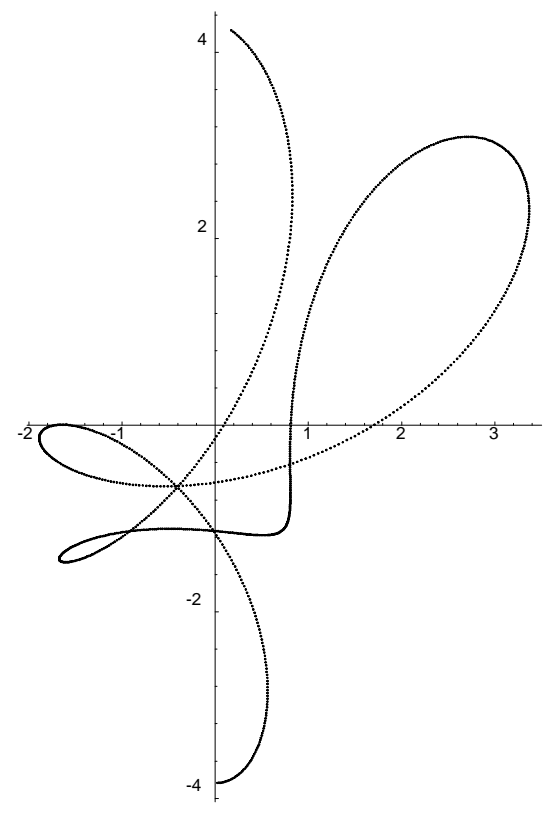

FiguRE 4. $\alpha=2 / 5$ for $50 \leq t \leq 60$

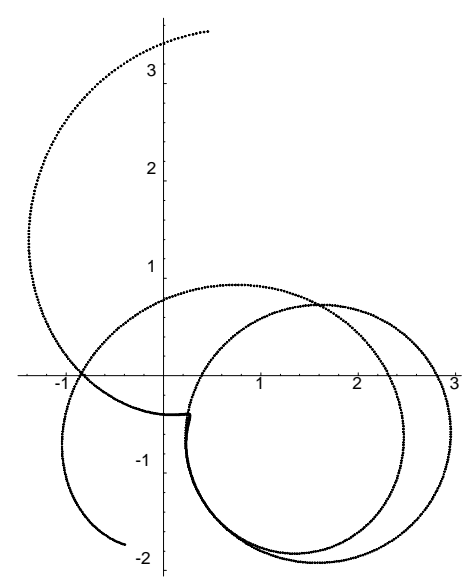

Figure $6 . \alpha=4 / 5$ for $50 \leq t \leq 60$

(The horizontal and vertical axes indicate the real and imaginary parts of the Hurwitz zeta function.) 
The Dirichlet L-function: $L(1 / 2+i t, \chi)$.

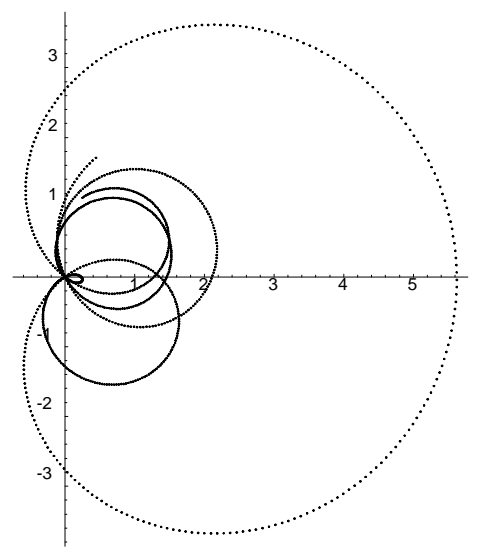

Here $\chi$ is the Dirichlet character mod 5 determined by $\chi(2)=\exp \left(2 \pi i \frac{3}{4}\right)$. Hence, we have

$$
L(s, \chi)=5^{-s} \sum_{j=1}^{4} \chi(j) \zeta(s, j / 5) .
$$

FiguRE $7.50 .00 \leq t \leq 60$

5.2. $L$-function of $E_{11}: L\left(1+i t, E_{11}\right)$

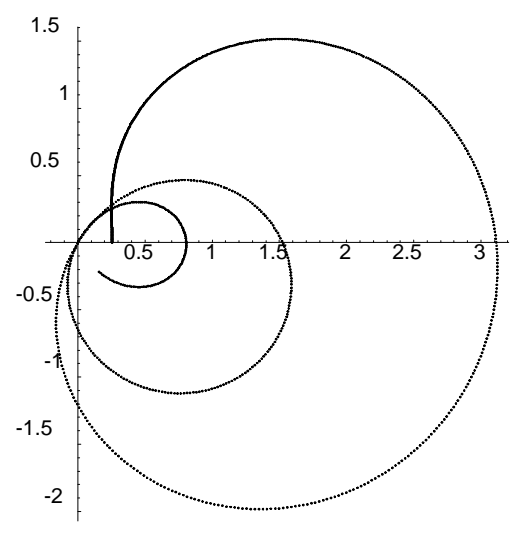

Figure $8.0 .00 \leq t \leq 9.89$

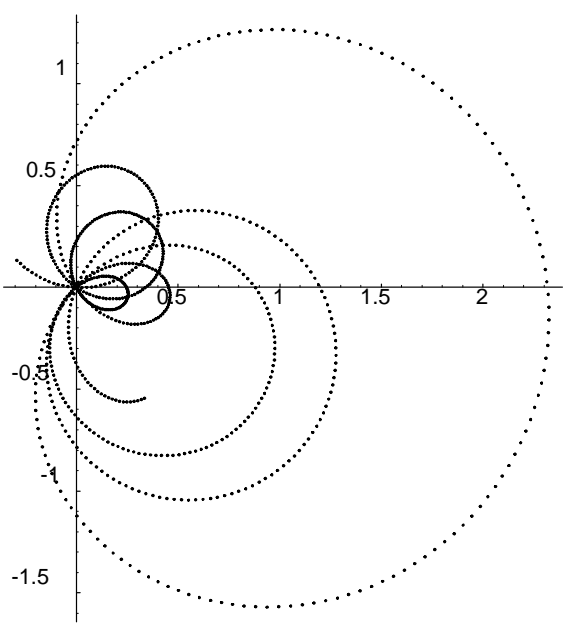

FiguRE $9.397 .00 \leq t \leq 401.00$

(The horizontal and vertical axes indicate the real and imaginary parts of the $L(1 / 2+i t, \chi)$ and $\left.\left(1+i t, E_{11}\right).\right)$ 
5.3. $L$-function of $E_{37}: L\left(1+i t, E_{37}\right)$.
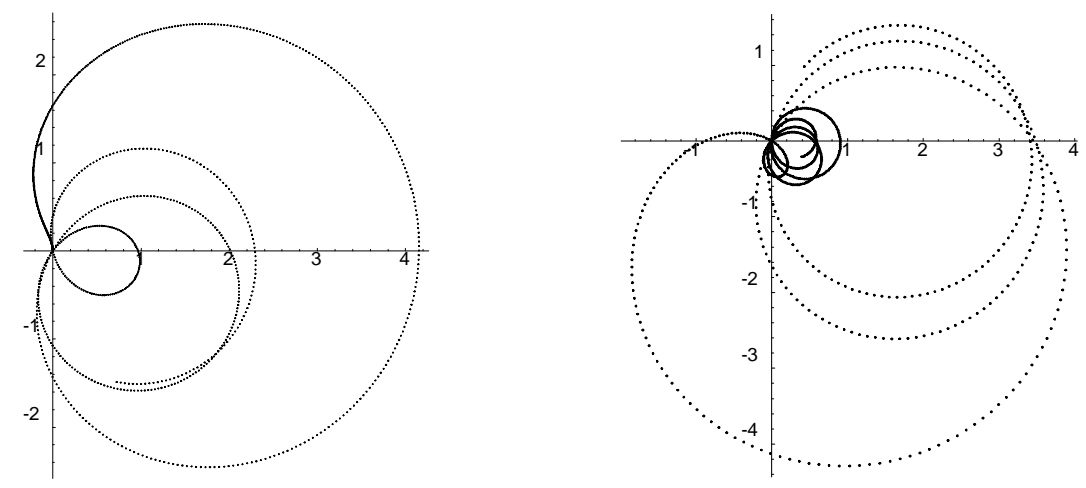

Figure $10.0 .00 \leq t \leq 9.39$

Figure 11. $396.03 \leq t \leq 400.00$

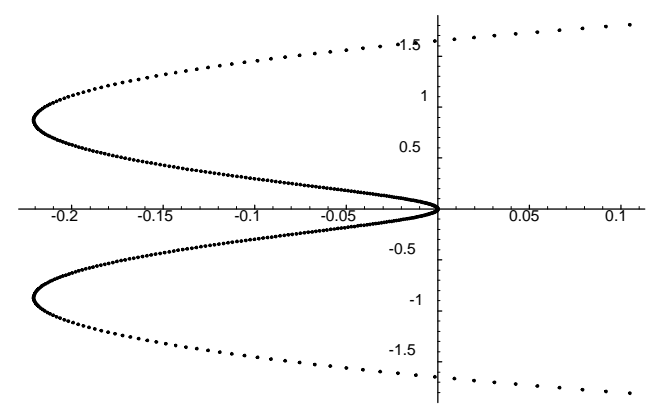

FIGURE $12 . \quad-1.99 \leq t \leq 1.99$

TABLE 1

$\begin{array}{cc}t & L\left(1+i t, E_{37}\right) \\ 0.000 & 0.000000000000000 \\ 0.001 & -0.000000186547781+0.000305999910626 i \\ 0.002 & -0.000000746190931+0.000612000642000 i \\ 0.003 & -0.000001678928871+0.000918003014876 i \\ 0.004 & -0.000002984760633+0.001224007850009 i \\ 0.005 & -0.000004663684865+0.001530015968161 i \\ 0.006 & -0.000006715699827+0.001836028190105 i \\ 0.007 & -0.000009140803393+0.002142045336623 i \\ 0.008 & -0.000011938993049+0.002448068228511 i \\ 0.009 & -0.000015110265897+0.002754097686581 i \\ 0.010 & -0.000018654618649+0.003060134531663 i \\ 0.011 & -0.000022572047631+0.003366179584607 i \\ 0.012 & -0.000026862548784+0.003672233666284 i \\ 0.013 & -0.000031526117659+0.003978297597591 i \\ 0.014 & -0.000036562749423+0.004284372199452 i \\ 0.015 & -0.000041972438852+0.004590458292819 i\end{array}$

(The horizontal and vertical axes indicate the real and imaginary parts of the $L\left(1+i t, E_{37}\right)$.) 
5.4. $L$-function of $E_{446}: L\left(1+i t, E_{446}\right)$.

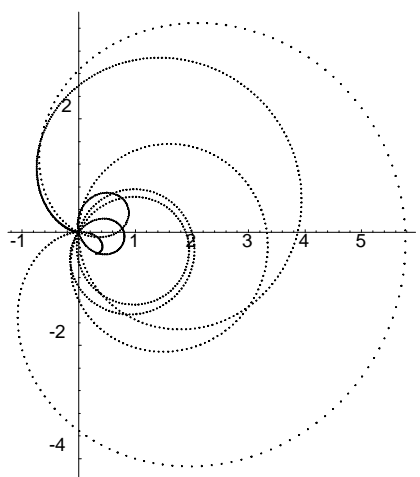

Figure $13.0 .00 \leq t \leq 9.39$

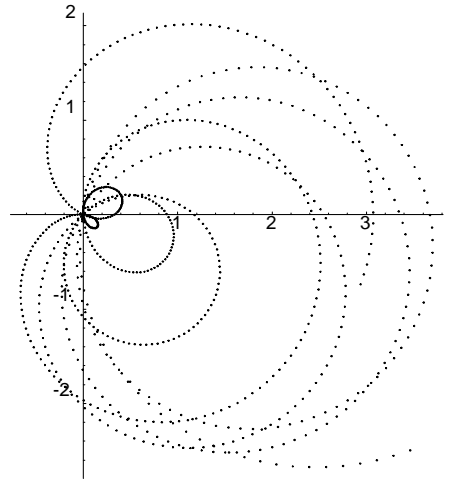

FiguRE 14. $396.03 \leq t \leq 400.00$

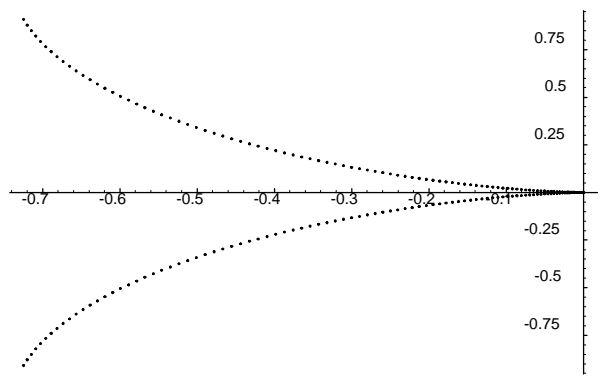

FiguRE $15 . \quad-1.99 \leq t \leq 1.99$

TABLE 2

$\begin{array}{cc}t & L\left(1+i t, E_{446}\right) \\ 0.000 & 0.000000000000000 \\ 0.001 & -0.000000940282805+0.000000000597143 i \\ 0.002 & -0.000003761133629+0.000000004777156 i \\ 0.003 & -0.000008462559692+0.000000016122982 i \\ 0.004 & -0.000015044573030+0.000000038217700 i \\ 0.005 & -0.000023507190494+0.000000074644603 i \\ 0.006 & -0.000033850433742+0.000000128987263 i \\ 0.007 & -0.000046074329248+0.000000204829601 i \\ 0.008 & -0.000060178908289+0.000000305755963 i \\ 0.009 & -0.000076164206951+0.000000435351183 i \\ 0.010 & -0.000094030266122+0.000000597200654 i \\ 0.011 & -0.000113777131493+0.000000794890405 i \\ 0.012 & -0.000135404853548+0.000001032007162 i \\ 0.013 & -0.000158913487567+0.000001312138424 i \\ 0.014 & -0.000184303093618+0.000001638872532 i \\ 0.015 & -0.000211573736555+0.000002015798735 i\end{array}$

(The horizontal and vertical axes indicate the real and imaginary parts of the $L\left(1+i t, E_{446}\right)$.) 


\section{5. $L$-function of $E_{5077}: L\left(1+i t, E_{5077}\right)$.}

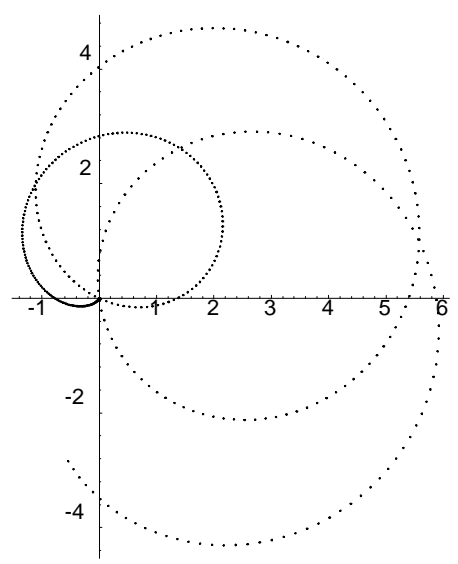

FigURE 16. $0 \leq t \leq 4.19$

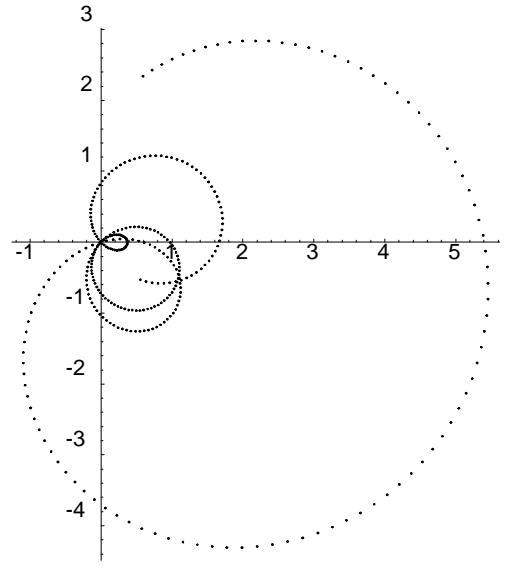

FiguRE $17.5 .00 \leq t \leq 8.39$

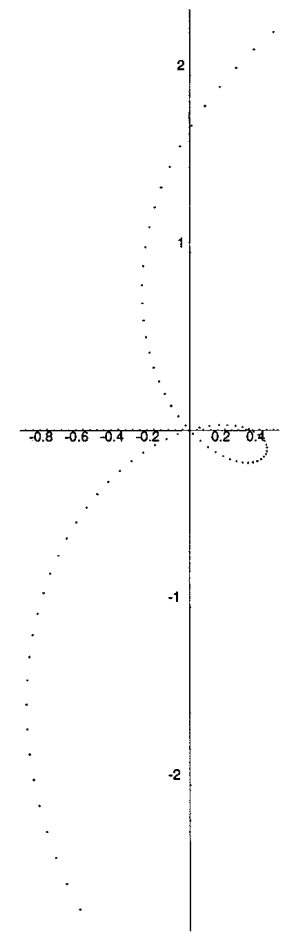

FIGURE $18.4 .20 \leq t \leq 4.99$

(The horizontal and vertical axes indicate the real and imaginary parts of the $L\left(1+i t, E_{5077}\right)$.) 


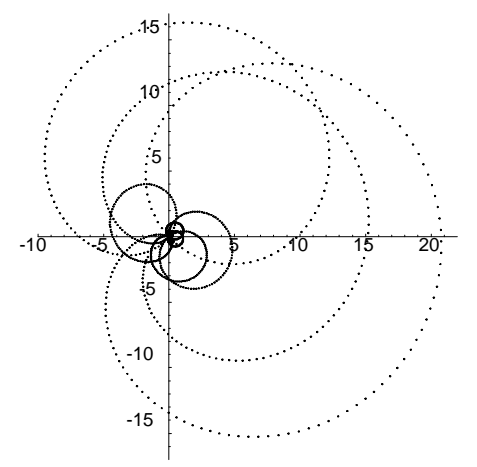

FigURE 19. $390.00 \leq t \leq 396.00$

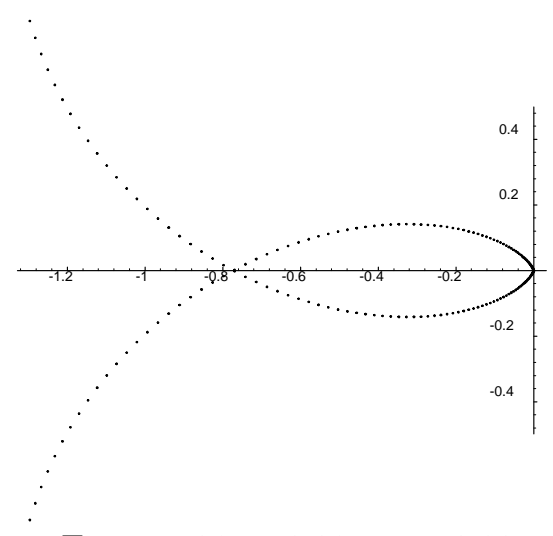

FiguRE $20 . \quad-0.99 \leq t \leq 0.99$

\section{TABLE 3}

\begin{tabular}{|c|c|}
\hline$t$ & $L\left(1+i t, E_{5077}\right)$ \\
\hline 0.000 & 0.000000000000000 \\
\hline 0.001 & $-0.000000000003206-0.000000001731847 i$ \\
\hline .002 & $-0.000000000051294-0.000000013854710 i$ \\
\hline 003 & $-0.000000000259678-0.000000046759267 i$ \\
\hline 004 & $-0.000000000820708-0.000000110835526 i$ \\
\hline 005 & $-0.000000002003678-0.000000216472487 i$ \\
\hline .006 & $-0.000000004154815-0.000000374057805 i$ \\
\hline 0.007 & $-0.000000007697282-0.000000593977454 i$ \\
\hline 0.008 & $-0.000000013131172-0.000000886615393 i$ \\
\hline 0.009 & $-0.000000021033507-0.000001262353229 i$ \\
\hline 010 & $32058228-0.000001731569878 i$ \\
\hline 011 & $-0.000000046936197-0.000002304641235 i$ \\
\hline 012 & $-0.000000066475186-0.000002$ \\
\hline 013 & $-0.000000091559873-0.000003803834498 i$ \\
\hline 0.014 & $-0.000000123151835-0.000004750690038 i$ \\
\hline 0.015 & $-0.000000162289539-0.000005842866880 i$ \\
\hline
\end{tabular}

(The horizontal and vertical axes indicate the real and imaginary parts of the $L\left(1+i t, E_{5077}\right)$.) 
Graphs of $L\left(\sigma+i t, E_{5077}\right)$ for various $\sigma$, and $17.7 \leq t \leq 20.045$.

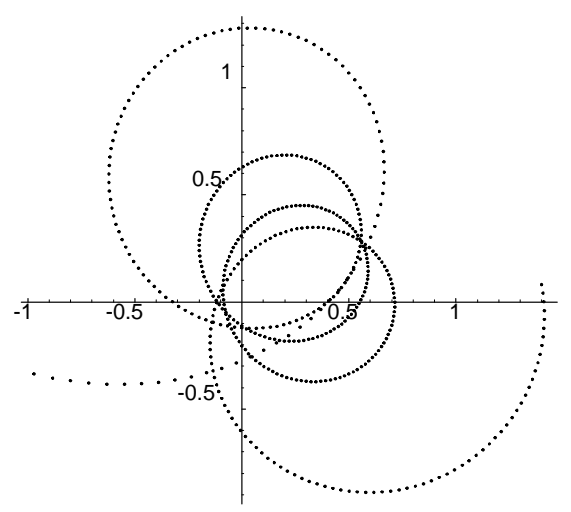

Figure 21. $\sigma=0.98$

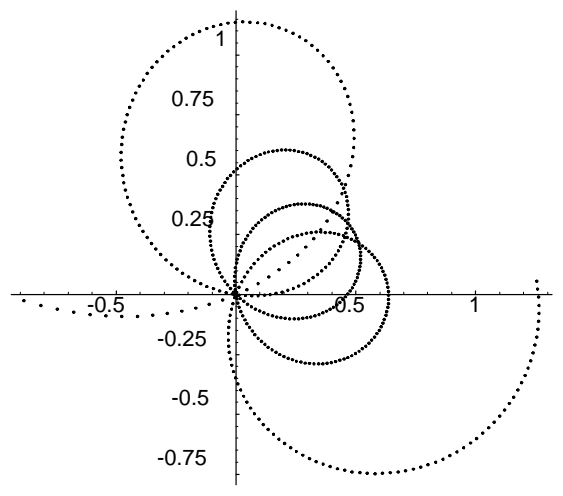

Figure 22. $\sigma=1.00$

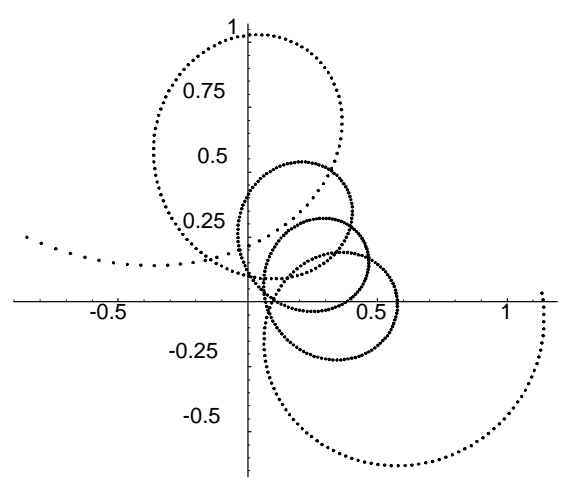

FiguRE 23. $\sigma=1.02$

(The horizontal and vertical axes indicate the real and imaginary parts of the $L\left(\sigma+i t, E_{5077}\right)$.) 


\subsection{Numerical experiment of Conjecture 1.}

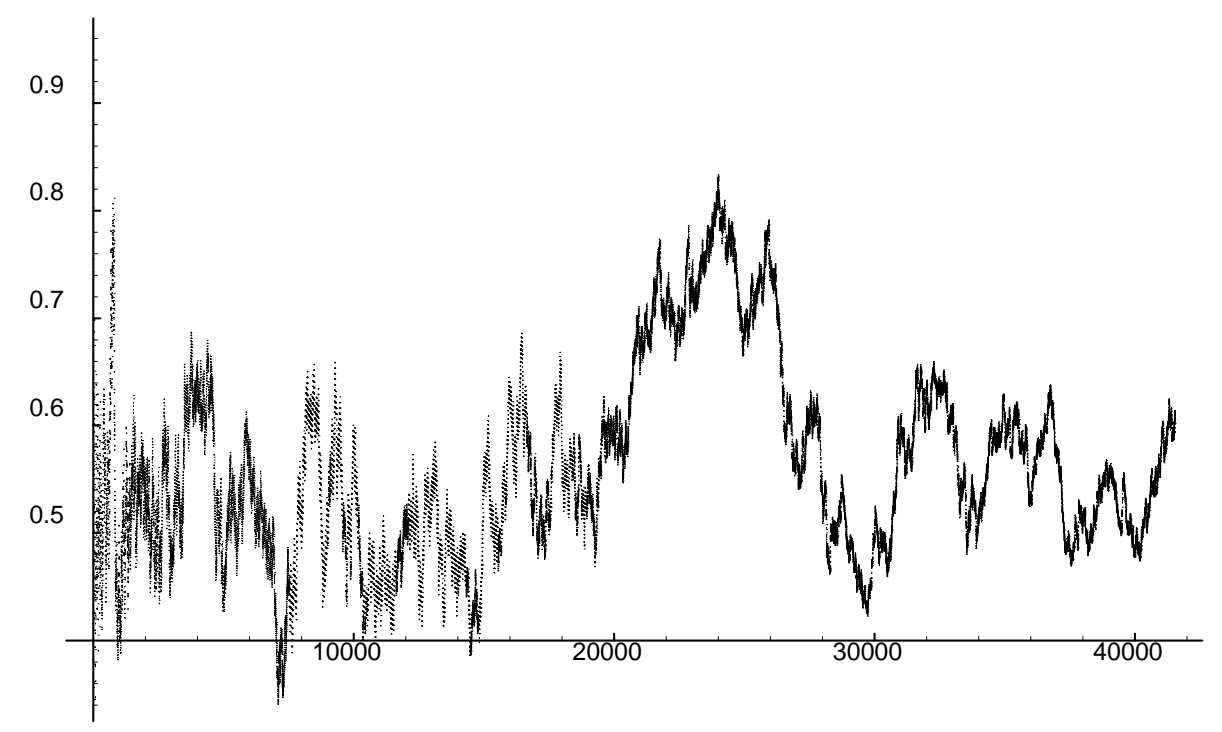

FIGURE 24. Discrepancy for $E_{11}$

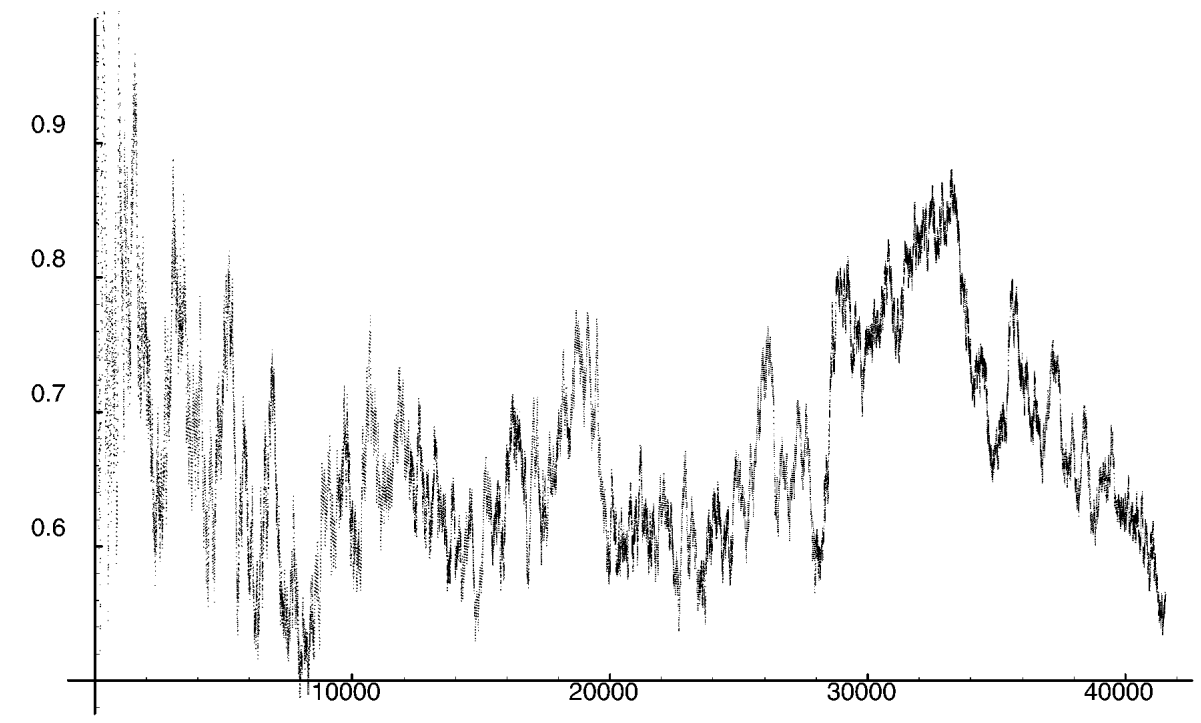

FiguRE 25. Discrepancy for $E_{37}$

(The horizontal and vertical axes indicate $K$ and $\sqrt{K} D_{K}^{(S T)}\left(x_{n}\right)$, respectively.) 


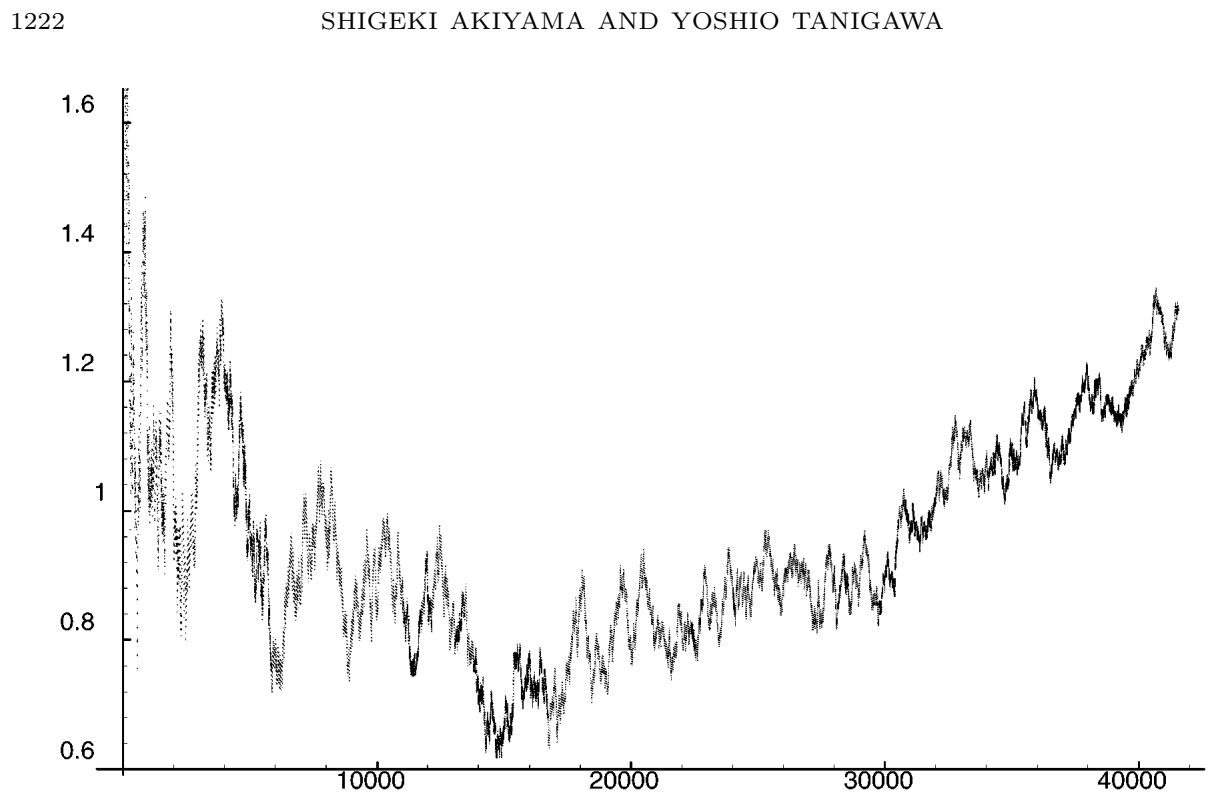

FiguRE 26. Discrepancy for $E_{446}$

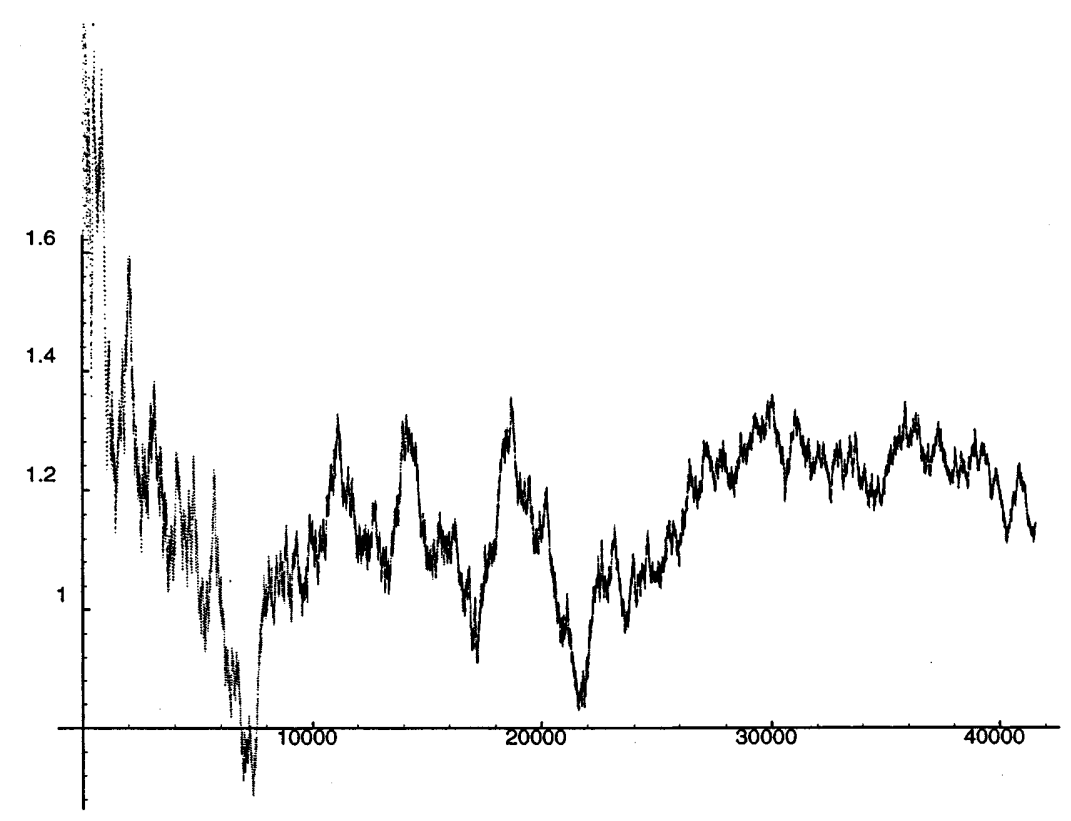

FiguRE 27. Discrepancy for $E_{5077}$

(The horizontal and vertical axes indicate $K$ and $\sqrt{K} D_{K}^{(S T)}\left(x_{n}\right)$, respectively.) 
5.7. Zeros of $L$-functions. In Tables 4, 5, 6 and 7, we list the imaginary parts of zeros of $L$-functions. All zeros we have found are of the form $s=1+i t$.

TABLE 4. Zeros of $L\left(s, E_{11}\right), \quad \operatorname{Im}(s) \leq 400$

\begin{tabular}{|c|c|c|c|c|c|c|c|c|c|c|c|}
\hline 6.363 & .604 & & & 3.569 & & & & & & & \\
\hline 25.210 & 25.876 & & & & & & & & & & \\
\hline 37.762 & & & & & & & & & & & \\
\hline 50.244 & & 52.256 & & & & & & 57.332 & & & \\
\hline & 62.339 & & & & & & & & & & \\
\hline 71.997 & & & & & & & & & & & \\
\hline 81.952 & 82.649 & 3.562 & & & & & & & & & \\
\hline & & & & & & & & & & & \\
\hline 101.499 & & & & & & & & & & & \\
\hline & & 12.151 & & & & & & & & & \\
\hline & & & & & & & & & & & \\
\hline & & & & & & & & & & & \\
\hline & & 38.968 & & & & & & & & & \\
\hline & & & & & & & & & & & \\
\hline & & & & & & & & & & & \\
\hline & 64.288 & & & & & & & & & & \\
\hline & & & & & & & & & & & \\
\hline & & & & & & & & & & & \\
\hline & & & & & & & & & & & \\
\hline & & & & & & & & & & & \\
\hline & & & & & & & & & & & \\
\hline & & & & & & & & & & & \\
\hline & & & & & & & & & & & \\
\hline & & & & & & & & & & & \\
\hline 36.316 & 45 & 22 & & & & & & & & & \\
\hline & & & & & & & & & & & \\
\hline & & & & & & & & & & & \\
\hline & & & & & & & & & & & \\
\hline & & & & & & & & & & & \\
\hline & & & & & & & & & & & \\
\hline & & & & & & & & & & & \\
\hline & & & & & & & & & & & \\
\hline & & & & & & & & & & & \\
\hline & & & & & & & & & & & \\
\hline & & & & & & & & & & & \\
\hline & & & & & & & & & & & \\
\hline & & & & & & & & & & & \\
\hline & & & & & & & & & & & \\
\hline & & & & & & & & & & & \\
\hline & & & & & & & & & & & \\
\hline & & & & & & & & & & & \\
\hline & & & & & & & & & & & \\
\hline & & & & & & & & & & & \\
\hline 7.894 & & 32 & & 380 & & & & & & & \\
\hline & & & & & & & & & & & \\
\hline & & & & & & & & & 397. & & \\
\hline 399.005 & 399.619 & 399.952 & 400 & & & & & & & & \\
\hline
\end{tabular}


TABLE 5. Zeros of $L\left(s, E_{37}\right), \quad \operatorname{Im}(s) \leq 400$

\begin{tabular}{|c|c|c|c|c|c|c|c|c|c|c|c|}
\hline 0.000 & 5.003 & .870 & 8.014 & 9.933 & 10.775 & 11.757 & 12.958 & 15.604 & 16.192 & 17.142 & 18.064 \\
\hline 18.787 & 19.815 & 21.323 & 22.620 & 23.328 & 24.169 & 25.657 & 26.814 & 27.339 & 28.190 & 29.030 & \\
\hline 30.896 & 32.042 & 33.441 & & & 35.462 & & & & & & \\
\hline 41.652 & 42.575 & 44.034 & 44.236 & 45.393 & 45.565 & 46.653 & 46.957 & 47.574 & 49.342 & 50.219 & \\
\hline & & & & & & & & & & & \\
\hline 61.445 & 62.295 & 62.640 & & 63.884 & 64.344 & 65.379 & & 67.215 & & & 9.527 \\
\hline 70.195 & 70.817 & 71.458 & 72.700 & 73.278 & 73.751 & 74.154 & 74.865 & 75.524 & 76.441 & 77.756 & \\
\hline 79.152 & 79.788 & & & & & & & & & & \\
\hline 87.315 & & & & & & & & & & & 95.292 \\
\hline 96.414 & 96.816 & 97.553 & 97.887 & 98.417 & & 99.767 & & 101.328 & 102.155 & 102.361 & 103.247 \\
\hline 104.001 & 104.763 & 105.428 & 106.597 & 107.276 & & & & & & & \\
\hline & & & & & & & & & & & \\
\hline 120.065 & 120.692 & 120.963 & 122.027 & 122.793 & 123.911 & 124.659 & & & & & \\
\hline 127.863 & 28.624 & 129. & & 130.774 & & & & & & & \\
\hline & & & & & & & & & & & \\
\hline 43.677 & 144.192 & 144. & & & & & & & & & \\
\hline & 51.976 & 152 & & 153 & & & & & & & \\
\hline & & & & & & & & & & & \\
\hline 65.844 & 166.576 & 167. & 167.572 & 168. & & & & & & & \\
\hline 73.007 & 173.852 & 174 . & & 176 & & & & & & & \\
\hline & & & & & & & & & & & \\
\hline & & & & & & & & & & & \\
\hline & & & & & & & & & & & \\
\hline & 30 & & & & & & & & & & \\
\hline & 10.121 & & & & & & & & & & \\
\hline & & & & & & & & & & & \\
\hline & 4 & & & & & & & & & & \\
\hline & 30.945 & 85 & 232.9 & 96 & & & & & & & \\
\hline & & & & & & & & & & & \\
\hline & 22 & & & & & & & & & & \\
\hline & 52.056 & & & & & & & & & & \\
\hline & & & & & & & & & & & \\
\hline 29 & & & & & & & & & & & \\
\hline 664 & 72.5 & & & & & & & & & & \\
\hline 278.988 & 279.494 & & & & & & & & & & \\
\hline & & & & & & & & & & & \\
\hline & 12 & & & & & & & & & & \\
\hline 298.553 & & & & & & & & & & & \\
\hline & & & & & & & & & & & \\
\hline & 15 & & & & & & & & & & \\
\hline 318.542 & 319.328 & 319.6 & 320.074 & & & & & & & 410 & \\
\hline & & & & & & & & & & & \\
\hline & & & & & & & & & & & \\
\hline 38.046 & 38.370 & 90 & & 340.2 & 341. & & & & & 651 & 059 \\
\hline & & & & & & & & & & & \\
\hline & & & & & & & & & & & \\
\hline 57.650 & 5 & & & & 360 & 360 & & 361 & & & \\
\hline & & & & & & & & & & & \\
\hline & & & & & & & & & & & \\
\hline 77.159 & 77.534 & 377.954 & & & & & 380. & & & 382.267 & 382. \\
\hline 83.045 & 83.417 & 67 & 384.626 & 385.331 & 300.1 & 386.948 & 387. & 387.8 & & & 389. \\
\hline & & & & & & & & & 394.349 & 394.915 & \\
\hline & & & & & & & & & & & \\
\hline
\end{tabular}


TABLE 6 . Zeros of $L\left(s, E_{446}\right), \quad \operatorname{Im}(s) \leq 400$

\begin{tabular}{|c|c|c|c|c|c|c|c|c|c|c|c|}
\hline 0.000 & 3.017 & 4.440 & 5.480 & 6.649 & 7.361 & 8.060 & 9.759 & 10.227 & 11.122 & 11.943 & .387 \\
\hline 13.046 & 15.042 & 15.644 & 16.306 & 16.548 & 17.516 & 18.552 & 18.851 & 19.701 & 20.271 & 21.663 & \\
\hline 23.002 & 23.421 & 24.000 & 24.720 & 26.117 & 26.489 & 27.238 & 27.793 & 28.535 & 28.872 & & \\
\hline 31.285 & 31.977 & 32.376 & 33.782 & 34.081 & 34.548 & 35.207 & 35.576 & 36.140 & 36.906 & 38.247 & \\
\hline & & & & & & & & & & & \\
\hline 46.609 & 46.980 & 47.504 & 48.280 & 49.272 & 50.095 & 50.573 & 51.286 & 51.564 & 52.062 & 52.433 & \\
\hline 54.306 & 54.540 & 55.299 & 55.708 & 56.325 & 57.211 & 57.686 & 58.071 & 58.601 & 58.893 & 60.128 & 60.999 \\
\hline & & 62.382 & 63.011 & & & & & & & & \\
\hline 68.238 & 68.682 & 69.396 & 70.124 & 70.515 & & 71.288 & & & & & \\
\hline 74.736 & 75.360 & 75.878 & 76.665 & 77.697 & 78.243 & 78.484 & 78.891 & 79.765 & 80.376 & 80.885 & 112 \\
\hline 81.525 & 82.057 & 83.000 & 83.498 & 84.108 & 84.646 & 85.267 & 85.897 & & & & \\
\hline 88.583 & 89.525 & 89.729 & 90.363 & 90.593 & & & & & & & \\
\hline 94.992 & 96.097 & 96.529 & 96.891 & 97.287 & 97.757 & 98.087 & 98.549 & & & & \\
\hline 101.891 & 101.926 & 102.705 & 102.874 & 103.480 & 104.234 & 104.849 & 105.253 & & & & \\
\hline & 108.589 & 09.210 & 109.556 & 63 & & 110.5 & & 97 & & & \\
\hline 4.170 & 115.042 & 15.542 & 115.778 & 71 & & 117.542 & & & & & \\
\hline 120.435 & 120.751 & 121.532 & 122.076 & 122. & 123.415 & 124.087 & & & & & \\
\hline & 127.407 & & 128.385 & & & & & & & & \\
\hline 132.891 & 133.481 & & 134.905 & & & & & & & & \\
\hline 138.847 & 139.801 & 140. & 141.134 & 141. & 14 & 142. & 142 . & & & & \\
\hline 144.972 & 145.659 & 146. & 147.226 & 147 & & 148. & & & & & \\
\hline & 152.164 & 49 & 152.846 & & & 154 & & & & & \\
\hline 157.310 & 158.014 & & 159. & & & & & & & & \\
\hline 163.864 & 164.094 & 49 & 164 & 12 & & 166. & 88 & & & & \\
\hline & 170.319 & & & & & & & & & & \\
\hline 175.515 & 175.908 & 176.242 & 176.949 & & & & & & & & \\
\hline 181.220 & 181.6 & & 182 & & & & 89 & & & & \\
\hline & & & & & & & & & & & \\
\hline & 193.5 & & & & & & & & & & \\
\hline 98.856 & 199.355 & & 200 & & 52 & 70 & 44 & 94 & & & \\
\hline & 205.276 & & & & & & & & & & \\
\hline 210.361 & 210.652 & 11.193 & 211.508 & 3 & 212.719 & 212.839 & 213.678 & & & & \\
\hline 06 & 16.6 & & 217 & & & & & & & & \\
\hline 222.163 & 222.497 & & & & & & & & & & \\
\hline & 227.852 & 87 & 228.673 & & 229.784 & & 30.520 & & & & \\
\hline & 33.772 & & & & & & & & & & \\
\hline & 238.971 & & 240 & & & & & & & & \\
\hline & 244.979 & & & & & & & & & & \\
\hline & & & & & & & & & & & \\
\hline & 256.2 & & 257 & & & & & & & & \\
\hline 261.400 & 261.938 & & 262.374 & & & & & & & & \\
\hline & & & & & & & & & & & \\
\hline & 6 & & & & & & & & & & \\
\hline 77.640 & 278.286 & & 279.188 & & & & 280 . & 281 & 281 & & 28 \\
\hline & & & & & & & & & & & \\
\hline & & & & & & & & & & & \\
\hline 293.919 & 294.407 & & & & & & & & & & 299 \\
\hline & & & & & & & & & & & \\
\hline & & & & & & & & & & & \\
\hline 0.410 & 310.796 & & & & & & & & & & \\
\hline & & & & & & & & & & & \\
\hline & & & & & & & & & & & \\
\hline & 327.058 & 327.640 & 328.008 & 5 & 328.778 & 36 & 329.961 & 330. & 330. & 96 & \\
\hline 332.348 & 332.561 & & & & & & & & & & \\
\hline & & & & & & & & & & & \\
\hline & & & & & & & & & & & \\
\hline .233 & 348.6 & & & & & & & & & & \\
\hline & & & & & & & & & & & \\
\hline & & & & & & & & & & & \\
\hline 364.125 & 364.634 & & 365.390 & & 366.552 & & 367.148 & 367.4 & 367.942 & 455 & \\
\hline & & & & & & & & & & & \\
\hline & & & & & & & & & & & \\
\hline 380.004 & 380.432 & 380.972 & 381.715 & 381.946 & 382.322 & 382.525 & 382.951 & 383.435 & 383.735 & 384.378 & 384.654 \\
\hline & 385.696 & 86.514 & & & & & & & & & \\
\hline & & & & & & & & & & & \\
\hline 90.0 & 96.257 & 396.707 & 397.181 & 397.6 & 398.083 & 98.408 & 399.082 & 399.269 & 399.566 & 400.335 & \\
\hline
\end{tabular}


TABLE 7. Zeros of $L\left(s, E_{5077}\right), \quad \operatorname{Im}(s) \leq 400$

\begin{tabular}{|c|c|c|c|c|c|c|c|c|c|c|c|}
\hline 0.000 & 2.052 & 3.262 & 4.471 & 4.754 & 6.012 & 6.623 & 7.343 & 7.707 & 8.477 & 9.382 & \\
\hline 10.496 & 11.033 & 11.687 & 12.287 & 12.973 & 13.152 & 14.942 & 15.515 & 15.895 & 16.440 & 16.643 & \\
\hline 18.073 & 18.560 & 19.031 & 19.497 & 19.975 & 20.660 & 21.759 & 22.216 & 22.735 & 23.224 & 23.721 & 24.030 \\
\hline 24.827 & 25.822 & 26.322 & 26.557 & 27.205 & 27.741 & 28.215 & 28.455 & 29.030 & 29.581 & 29.778 & \\
\hline 31.675 & 31.827 & 32.338 & 33.462 & 33.975 & 34.394 & 34.547 & 35.200 & & 35.817 & 36.427 & 5.913 \\
\hline 38.058 & 38.411 & 38.720 & 39.262 & 39.513 & 39.965 & 40.721 & 41.575 & 41.816 & 42.524 & 42.670 & 3.755 \\
\hline 44.262 & 44.430 & 44.988 & 45.323 & 46.028 & 46.336 & 46.743 & 46.999 & & 48.029 & & \\
\hline 50.192 & 50.521 & 51.132 & 51.475 & 51.811 & 52.379 & 52.575 & 53.526 & 53.922 & & & \\
\hline 55.633 & & & 57.229 & & & & & & & 61.152 & \\
\hline 61.905 & 62.192 & 62.406 & 63.165 & 63.346 & 63.757 & 64.193 & 64.662 & 65.336 & 65.719 & 66.272 & 66.712 \\
\hline 67.463 & 67.915 & 68.274 & 68.546 & 69.163 & 70.023 & 70.303 & 70.709 & 70.817 & & & \\
\hline 73.211 & 73.440 & 73.865 & 74.169 & 74.580 & 74.871 & 75.306 & 76.001 & 76.623 & 77.210 & 77.917 & \\
\hline 78.690 & 78.916 & 79.613 & 80.315 & 80.428 & 80.958 & 81.212 & 81.4 & 82.004 & 82.312 & 83.197 & \\
\hline 83.863 & & & & 85.895 & & 86.683 & & & & & \\
\hline 89.845 & 90.003 & & & & & & & & & & \\
\hline 94.877 & 95.529 & & 96.530 & 97.149 & 97.3 & 97.453 & 98.1 & 98.340 & 98.664 & 99.5 & 956 \\
\hline 100.227 & 0.712 & 09 & 101.820 & 29 & 102.3 & 102.809 & 16 & 666 & & & \\
\hline & 06.246 & & 07.046 & & & & & 94 & & & \\
\hline & 10.800 & 12.206 & 12.571 & 12.769 & & & & 114.532 & & & \\
\hline & 16.859 & 17.188 & 17.459 & 43 & 13 & 118. & 41 & 119.877 & & & \\
\hline & 121.663 & & 122.542 & & & & & & & & \\
\hline 6.354 & 126.873 & 127.424 & 128.158 & 128.229 & 128.689 & 128.945 & 129.619 & 130.022 & & 130.997 & \\
\hline 31.910 & 132.311 & 32.659 & 919 & & & & & 135. & & & \\
\hline & 137.173 & & & & & & & & & & \\
\hline 142.128 & 142.407 & 143.306 & 143.556 & & & 144.527 & 144.799 & 145.156 & 145.729 & & \\
\hline .167 & 617 & & 98 & & & & & & & & \\
\hline & 152.650 & & & & & & & & & & \\
\hline 57.083 & 157.577 & 58.386 & 158.597 & & & 159.987 & 160.1 & 160.600 & & & \\
\hline 62.114 & 658 & & 163 & & & & & & & & \\
\hline & 167.745 & & & & & & & & & & \\
\hline 172.308 & 172.433 & 172.971 & 173.168 & 173.785 & 174.332 & 174.782 & 175.244 & 175.632 & & 176.465 & \\
\hline 77.184 & 177 & & & & & & & & & & \\
\hline & 182.811 & & & & & & & & & & \\
\hline & 187.856 & & 455 & & & & 190.1 & & & & \\
\hline & 192 & & & & & & & & & & \\
\hline & 197.475 & & & & & & & & & & \\
\hline 1.725 & 202.128 & 2.388 & 203 & & & & 204. & 205. & & & \\
\hline & 206.966 & & & & & & & & & & \\
\hline & 212.089 & & & & & & & & & & \\
\hline 216.615 & 216.870 & 217.362 & 217.590 & 217.945 & 218.270 & 91 & 218.922 & 219.522 & 219.779 & 64 & 46 \\
\hline & & & & & & & & & & & \\
\hline & 403 & & & & & & & & & & \\
\hline 30.497 & 230.896 & 31.502 & 232.491 & & & & & 234 . & & & \\
\hline & & & & & & & & & & & \\
\hline & 8 & & & & & & & & & & \\
\hline & 245.588 & & 246.271 & & 246 & & 247.7 & 54 & & & \\
\hline & & & & & & & & & & & \\
\hline & & & & & & & & & & & \\
\hline 9.111 & 259.704 & 260.432 & 260.841 & 61.353 & 261.5 & & 262.1 & 262.3 & 83 & & \\
\hline & & & & & & & & & & & \\
\hline & & & & & & & & & & & \\
\hline & 73.850 & & & & & & & & & & \\
\hline & & & & & & & & & & & \\
\hline & & & & & & & & & & & \\
\hline 37.573 & & & & & & & & & & & \\
\hline & & & & & & & & & & & \\
\hline & & & & & & & & & & & \\
\hline & 35 & & & & & & & & & & \\
\hline 306.157 & & & & & & & & & & & \\
\hline & & & & & & & & & & & \\
\hline & & & & & & & & & & & \\
\hline 319.816 & 319.997 & 320.451 & 320.796 & & & & & & & & 328 \\
\hline & & & & & & & & & & & \\
\hline & & & & & & & & & & & \\
\hline 333.357 & 34.158 & & & & & & & 336. & 20 & & 337.713 \\
\hline & & & & & & & & & & & \\
\hline & & & & & & & & & & & \\
\hline .496 & 347.81 & & & & & & & 350. & & & \\
\hline & 352.384 & & & & & & & & & & \\
\hline & & & & & & & & & & & \\
\hline 0.984 & 361.427 & 361.7 & 362.120 & 362.4 & & 19 & 363.6 & 364. & 331 & & \\
\hline & & & & & & & & & & & \\
\hline & & & & & & & & & & & \\
\hline & & & & & & & 377.3 & 377.5 & & & 378.744 \\
\hline .973 & 379.460 & 379.6 & 380.1 & 6 & 381.0 & 381.5 & 381.8 & 382.1 & 382.347 & & \\
\hline & & & & & & & & & & & \\
\hline & & & & & & & & & & & \\
\hline & & 393 & 93.899 & 4.049 & 994. & 94.658 & 395.074 & 395.232 & 96.055 & 396.243 & 396.980 \\
\hline
\end{tabular}

$\begin{array}{lllllllllll}397.112 & 397.387 & 397.861 & 398.324 & 398.687 & 398.998 & 399.135 & 399.527 & 399.926 & 400.512\end{array}$ 


\section{Appendix: Type SeQuence And Rosser's LaW}

In the history of calculation of zeros of the Riemann zeta function, two laws, which are not exact laws, have appeared that play an important role. They are Gram's and Rosser's laws and formulated as follows. First we recall that the argument of $\zeta(1 / 2+i t)$ is given by $-\vartheta(t)$, where

$$
\vartheta(t)=\operatorname{Im}\left(\log \Gamma\left(\frac{1}{4}+i \frac{t}{2}\right)\right)-\frac{1}{2} t \log \pi .
$$

Let $g_{k}$ be the positive real number which satisfies

$$
\vartheta\left(g_{k}\right)=k \pi, \quad k=-1,0,1,2, \cdots
$$

These numbers $g_{k}$ are called ( $k$-th) Gram points. Gram's law is stated as follows.

Gram's law: There exists exactly one zero in the interval $\left(1 / 2+i g_{k}, 1 / 2+i g_{k+1}\right)$.

This statement is slightly stronger than usual in the sense that it asserts "exactly one" instead of "at least one". To simplify matters, we assume that $\zeta\left(1 / 2+i g_{k}\right) \neq 0$ for any Gram point $g_{k}$. If $\zeta\left(1 / 2+i g_{k}\right)>0$ (resp. $\left.<0\right)$, then $g_{k}$ is called a good (resp. bad) Gram point. If all the Gram points are good then Gram's law is true. But this is not the case. This law fails for the first time at $g_{126}=282.454 \cdots$. In fact, $\zeta(1 / 2+i t) \neq 0$ for $g_{125}<t<g_{126}$, and $g_{126}$ is a bad Gram point.

Define a Gram block of length $k$ by the set of consecutive Gram points $B_{n}=$ $\left\{g_{n}, g_{n+1}, \ldots, g_{n+k}\right\}$, where $g_{n}$ and $g_{n+k}$ are good and $g_{n+j}(j=1,2, \ldots, k-1)$ are bad. Then Rosser's law is stated as follows.

Rosser's law: Let $B_{n}=\left\{g_{n}, g_{n+1}, \ldots, g_{n+k}\right\}$ be a Gram block of length $k$, then $\zeta(s)$ has at least $k$ zeros in the interval $\left(1 / 2+i g_{n}, 1 / 2+i g_{n+k}\right)$.

This law fails for the first time at $B_{13999525}$.

Now we classify all the non-trivial zeros of $\zeta(s)$ into five classes.

Definition 1. Let $u=1 / 2+i t_{0}(t>0)$ be a zero of $\zeta(s)$. Define the zero $u$ to be of type $j(j=1,2,3,4)$ when $-i \zeta^{\prime}(u)$ belongs to the $j$-th quadrant $R_{j}$. If $u$ is not in the above cases, we say that $u$ is of type 0 .

It seems that type 0 does not occur for the Riemann zeta function. But for the case of the $L$-function associated to an elliptic curve $E$ of Mordell-Weil rank greater than one, it actually happens on the critical line $\operatorname{Re}(s)=1$, because $L^{\prime}(1, E)=0$ (under the Birch and Swinnerton-Dyer conjecture for this curve). Let $\varepsilon$ be a small positive number. When $u=1 / 2+i t_{0}$ is of type $j$, then the orbit of $\zeta(1 / 2+i t)$ for $t \in\left(t_{0}-\varepsilon, t_{0}+\varepsilon\right)$ starts from $R_{j}$ and passes through the origin.

We define the type sequence by the sequence of types of zeros and Gram points (represented by G or B according to a good or a bad Gram point) arranged in increasing order. For example, in the middle of Table 8, one can find the sequence "G B24 G4". The meaning of this sequence is as follows. As $t$ is increasing, the Good Gram point $\left(=g_{125}\right)$, the Bad Gram point $\left(=g_{126}\right)$, zero of type 2, zero of type $\mathbf{4}$, the Good Gram point $\left(=g_{127}\right)$ and zero of type $\mathbf{4}$ occur in this order. Hence we see that $B_{125}=\left\{g_{125}, g_{126}, g_{127}\right\}$ is a Gram block of length 2 and contains 2 zeros. We mentioned above that Rosser's law fails at $B_{13999525}$. The type sequence about this point is "BGBG424G". The Table 8 suggests that:

1. Gram's law seems to be true in a certain average sense,

2. A bad Gram point occurs when the order of a zero and a Gram point is reversed. 
Let us consider the first point. Let $S(r)=\pi^{-1} \arg \zeta(1 / 2+i r)$ where the branch of $i \arg \zeta(s)=\operatorname{Im}(\log (\zeta(s)))$ is taken along the lines joining $2,2+i r$ and $2+i r, 1 / 2+i r$. It is known that the number of zeros in the rectangle $\{z \in \mathbf{C}: 0<\operatorname{Re}(z)<1,0<$ $\operatorname{Im}(z) \leq r\}$ is equal to

$$
\frac{r}{2 \pi} \log \left(\frac{r}{2 \pi}\right)-\frac{r}{2 \pi}+S(r)+O(1 / r) .
$$

We can easily see that

$$
\frac{g_{k}}{2 \pi} \log \left(\frac{g_{k}}{2 \pi}\right)-\frac{g_{k}}{2 \pi}=k+O\left(1 / g_{k}\right) .
$$

Hence, it would be plausible that

Conjecture 2. $S\left(g_{k}\right)=0$ for all the good Gram points $g_{k}$ except the ones with zero density.

Conjecture 2 has another heuristic explanation. Let $g_{k}$ be a "generic" good Gram point. Then we may assume that the value of $\zeta\left(1 / 2+i g_{k}\right)$ is greater than 1 (see Theorem 10.6 of [20]). Let $\sigma>1 / 2$ and $\delta$ be a small positive number. We compare the graphs of $\zeta(1 / 2+i r)$ and $\zeta(\sigma+i r)$ in the Gaussian plain for $r \in\left[g_{k}-\delta, g_{k}+\delta\right]$. When $\sigma$ is increasing, the orbit of the graph of $\zeta(\sigma+i r)$ moves to the outer normal direction by the Cauchy-Riemann relation. Note that the argument of $\zeta(1 / 2+i r)$ always decreases. Thus when $\sigma$ gets larger, we may expect that the graph of $\zeta(\sigma+i r)$ will naturally approach a single value

$$
\lim _{\sigma \rightarrow \infty} \zeta(\sigma+i r)=1 \text {. }
$$

In this process, the graph would not approach the origin, as $\zeta\left(1 / 2+i g_{k}\right)>1$. This suggests Conjecture 2 . Of course, in the above argument, we assume that there are no non-trivial zeros in $\left\{z \in \mathbf{C}: 1 / 2 \leq \operatorname{Re}(z),\left|\operatorname{Im}(z)-g_{k}\right| \leq \delta\right\}$.

If Conjecture 2 is true, we could formulate Rosser's law in a strengthened way:

\section{Each Gram block of length $k$ has exactly $k$ zeros.}

In fact, if a Gram block of length $k$ has more than $k$ zeros, then the front or rear Gram block may have length more than the number of zeros in it.

Now we will treat the second point. Consider relatively simple cases when a zero and its adjacent Gram point are reversed. When the graph of $\zeta(1 / 2+i r)$ moves a little along the real axis, then the changes

$$
3 G \rightarrow B 2, \quad G 4 \rightarrow 1 B
$$

occur in the type sequence. On the other hand, when the graph moves along the imaginary axis, the changes

$$
1 B \rightarrow 2 B, \quad B 2 \rightarrow B 1
$$

occur. For example, $G B 24 G 4$ or $G 31 B G 4$ in Table 8 are obtained from the normal sequence $G 3 G 4 G 4$ by the replacements in (29).

Let $T$ be the type sequence provided with Rosser's law (we treat here the "abstract" type sequence), and let $T^{\prime}$ be the type sequence generated from $T$ by the successive replacements of (29) and (30). Then it is easily seen that $T^{\prime}$ also satisfies Rosser's law. Thus Rosser's law, somewhat complicated at first glance, can be grasped in the context of Gram's law in the "average" sense.

Our argument is naturally extended to $L$-functions associated to elliptic curves. Tables 9 and 10 are the type sequence of the $L$-functions associated to $E_{11}$ and $E_{5077}$. 


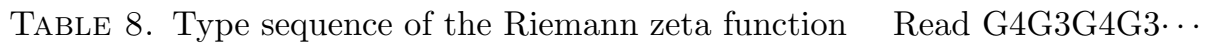

\begin{tabular}{|c|c|c|c|c|c|c|c|c|c|c|c|c|c|c|}
\hline G4 & G3 & G4 & G3 & G4 & G3 & G3 & G4 & G3 & G4 & G4 & G3 & G3 & G4 & G3 \\
\hline G4 & G4 & G4 & G3 & G4 & G4 & G3 & G4 & G3 & G4 & G3 & G3 & G4 & G4 & G3 \\
\hline G3 & G4 & G4 & G3 & G4 & G4 & G4 & G3 & G3 & G4 & $\mathrm{G} 4$ & G3 & G3 & G4 & G3 \\
\hline G4 & G3 & G3 & G4 & G4 & G3 & G4 & G3 & G4 & G4 & G3 & G3 & G4 & G4 & G4 \\
\hline G3 & G3 & G3 & G4 & G3 & G3 & G4 & G4 & G4 & G3 & G3 & G4 & G4 & G4 & G3 \\
\hline G4 & G3 & G4 & G4 & G3 & G3 & G3 & G4 & G3 & G3 & G3 & G3 & $\mathrm{G} 4$ & G4 & G3 \\
\hline G3 & G4 & G3 & G4 & G3 & G3 & G3 & G4 & $\mathrm{G} 4$ & G3 & G3 & G3 & $\mathrm{G} 4$ & G4 & G4 \\
\hline G3 & G3 & G4 & G4 & G4 & G3 & G4 & G3 & G4 & G4 & G3 & G3 & G4 & G4 & G4 \\
\hline G4 & G3 & G3 & G4 & G4 & G4 & $\mathrm{G}$ & B24 & G4 & G3 & G4 & G4 & G3 & G3 & G31 \\
\hline B & G4 & G3 & G3 & G4 & G3 & $\mathrm{G} 4$ & G4 & G3 & G3 & G4 & G4 & $\mathrm{G} 4$ & G3 & $\mathrm{G} 4$ \\
\hline G3 & G4 & $\mathrm{G} 4$ & G4 & G3 & G3 & $\mathrm{G} 4$ & G4 & G3 & G4 & G3 & G3 & G3 & $\mathrm{G} 4$ & $\mathrm{G} 4$ \\
\hline G3 & G3 & $\mathrm{G} 4$ & G3 & $\mathrm{G} 4$ & $\mathrm{G} 4$ & $\mathrm{G} 4$ & G3 & G3 & $\mathrm{G} 4$ & G4 & G4 & G3 & G3 & G3 \\
\hline G3 & G4 & $\mathrm{G} 4$ & G3 & G3 & G3 & G4 & G4 & G4 & G4 & G3 & G3 & G4 & G4 & G4 \\
\hline $\mathrm{G}$ & B23 & G4 & G3 & G4 & G4 & G3 & G3 & G3 & G4 & G4 & G3 & G3 & G3 & G3 \\
\hline G4 & G31 & B & G3 & G3 & G4 & G4 & G4 & G4 & G3 & G4 & G3 & G3 & G4 & G4 \\
\hline
\end{tabular}

TABle 9 . Type sequence for $L\left(s, E_{11}\right) \quad$ Read G3G3G4G4 $\cdots$

$\begin{array}{cllllllllllllll}\text { G3 } & \text { G3 } & \text { G4 } & \text { G4 } & \text { G4 } & \text { G3 } & \text { G3 } & \text { G4 } & \text { G4 } & \text { G4 } & \text { G3 } & \text { G4 } & \text { G3 } & \text { G3 } & \text { G3 } \\ \text { G31 } & \text { B } & \text { G4 } & \text { G3 } & \text { G3 } & \text { G3 } & \text { G3 } & \text { G4 } & \text { G3 } & \text { G4 } & \text { G4 } & \text { G4 } & \text { G4 } & \text { G3 } & \text { G3 } \\ \text { G } & \text { B24 } & \text { G4 } & \text { G4 } & \text { G4 } & \text { G4 } & \text { G3 } & \text { G3 } & \text { G3 } & \text { G4 } & \text { G3 } & \text { G4 } & \text { G4 } & \text { G4 } & \text { G4 } \\ \text { G4 } & \text { G3 } & \text { G3 } & \text { G3 } & \text { G3 } & \text { G4 } & \text { G4 } & \text { G41 } & \text { B } & \text { G4 } & \text { G3 } & \text { G4 } & \text { G3 } & \text { G3 } & \text { G3 } \\ \text { G3 } & \text { G4 } & \text { G3 } & \text { G31 } & \text { B } & \text { G4 } & \text { G3 } & \text { G4 } & \text { G } & \text { B23 } & \text { G3 } & \text { G4 } & \text { G4 } & \text { G4 } & \text { G4 } \\ \text { G4 } & \text { G4 } & \text { G3 } & \text { G3 } & \text { G3 } & \text { G3 } & \text { G3 } & \text { G3 } & \text { G4 } & \text { G41 } & \text { B } & \text { G4 } & \text { G4 } & \text { G4 } & \text { G3 } \\ \text { G3 } & \text { G3 } & \text { G3 } & \text { G3 } & \text { G31 } & \text { B } & \text { G3 } & \text { G4 } & \text { G3 } & \text { G4 } & \text { G4 } & \text { G3 } & \text { G } & \text { B23 } & \text { G3 } \\ \text { G3 } & \text { G4 } & \text { G4 } & \text { G4 } & \text { G4 } & \text { G4 } & \text { G3 } & \text { G4 } & \text { G3 } & \text { G3 } & \text { G3 } & \text { G4 } & \text { G3 } & \text { G3 } & \text { G4 } \\ \text { G41 } & \text { B } & \text { G4 } & \text { G3 } & \text { G3 } & \text { G3 } & \text { G } & \text { B23 } & \text { G3 } & \text { G4 } & \text { G4 } & \text { G3 } & \text { G4 } & \text { G4 } & \text { G3 } \\ \text { G4 } & \text { G4 } & \text { G3 } & \text { G3 } & \text { G3 } & \text { G3 } & \text { G3 } & \text { G3 } & \text { G4 } & \text { G31 } & \text { B } & \text { G4 } & \text { G3 } & \text { G4 } & \text { G } \\ \text { B23 } & \text { G3 } & \text { G4 } & \text { G4 } & \text { G3 } & \text { G4 } & \text { G4 } & \text { G4 } & \text { G4 } & \text { G4 } & \text { G4 } & \text { G3 } & \text { G3 } & \text { G3 } & \text { G3 } \\ \text { G3 } & \text { G3 } & \text { G3 } & \text { G4 } & \text { G41 } & \text { B } & \text { G4 } & \text { G3 } & \text { G4 } & \text { G3 } & \text { G3 } & \text { G3 } & \text { G3 } & \text { G3 } & \text { G4 } \\ \text { G3 } & \text { G4 } & \text { G4 } & \text { G41 } & \text { B } & \text { G3 } & \text { G4 } & \text { G3 } & \text { G3 } & \text { G } & \text { B23 } & \text { G3 } & \text { G4 } & \text { G4 } & \text { G4 } \\ \text { G4 } & \text { G4 } & \text { G4 } & \text { G3 } & \text { G3 } & \text { G4 } & \text { G4 } & \text { G3 } & \text { G3 } & \text { G3 } & \text { G3 } & \text { G3 } & \text { G4 } 4 & \text { G4 } & \text { G41 } \\ \text { B } & \text { G3 } & \text { G4 } & \text { G3 } & \text { G3 } & \text { G3 } & \text { G3 } & \text { G3 } & \text { G4 } & \text { G4 } & \text { G4 } & \text { G4 } & \text { G4 } & \text { G4 } & \text { G4 }\end{array}$

TABle 10 . Type sequence of $L\left(s, E_{5077}\right) \quad$ Read 0BG4G4 $\cdots$

$\begin{array}{clclllllllllllc}0 & \text { B } & \text { G4 } & \text { G4 } & \text { G3 } & \text { G4 } & \text { G3 } & \text { G3 } & \text { G3 } & \text { G4 } & \text { G4 } & \text { G3 } & \text { G3 } & \text { G4 } & \text { G4 } \\ \text { G4 } & \text { G4 } & \text { G41 } & \text { B } & \text { G } & \text { B2 } & \text { B23 } & \text { G3 } & \text { G4 } & \text { G4 } & \text { G3 } & \text { G4 } & \text { G4 } & \text { G41 } & \text { B } \\ \text { G4 } & \text { G3 } & \text { G3 } & \text { G3 } & \text { G3 } & \text { G41 } & \text { B } & \text { G4 } & \text { G } & \text { B2 } & \text { B23 } & \text { G3 } & \text { G3 } & \text { G3 } & \text { G4 } \\ \text { G4 } & \text { G42 } & \text { B } & \text { G4 } & \text { G3 } & \text { G4 } & \text { G4 } & \text { G } & \text { B2 } & \text { B2 } & \text { B24 } & \text { G3 } & \text { G31 } & \text { B1 } & \text { B1 } \\ \text { B } & \text { G } & \text { B23 } & \text { G4 } & \text { G41 } & \text { B1 } & \text { B } & \text { G4 } & \text { G3 } & \text { G4 } & \text { G3 } & \text { G4 } & \text { G } & \text { B2 } & \text { B23 } \\ \text { G3 } & \text { G4 } & \text { G3 } & \text { G4 } & \text { G41 } & \text { B1 } & \text { B1 } & \text { B } & \text { G3 } & \text { G3 } & \text { G } & \text { B23 } & \text { G } & \text { B23 } & \text { G4 } \\ \text { G31 } & \text { B } & \text { G3 } & \text { G3 } & \text { G3 } & \text { G3 } & \text { G4 } & \text { G41 } & \text { B } & \text { G3 } & \text { G4 } & \text { G3 } & \text { G4 } & \text { G41 } & \text { B } \\ \text { G3 } & \text { G4 } & \text { G } & \text { B1 } & \text { B2 } & \text { B23 } & \text { G4 } & \text { G3 } & \text { G4 } & \text { G41 } & \text { B1 } & \text { B } & \text { G4 } & \text { G4 } & \text { G4 } \\ \text { G4 } & \text { G3 } & \text { G3 } & \text { G3 } & \text { G4 } & \text { G4 } & \text { G } & \text { B23 } & \text { G31 } & \text { B } & \text { G4 } & \text { G3 } & \text { G } & \text { B2 } & \text { B23 } \\ \text { G4 } & \text { G4 } & \text { G41 } & \text { B1 } & \text { B } & \text { G4 } & \text { G4 } & \text { G3 } & \text { G } & \text { B23 } & \text { G3 } & \text { G4 } & \text { G3 } & \text { G } & \text { B23 } \\ \text { G3 } & \text { G41 } & \text { B } & \text { G41 } & \text { B } & \text { G3 } & \text { G3 } & \text { G4 } & \text { G4 } & \text { G3 } & \text { G3 } & \text { G3 } & \text { G4 } & \text { G41 } & \text { B } \\ \text { G3 } & \text { G3 } & \text { G } & \text { B2 } & \text { B2 } & \text { B23 } & \text { G3 } & \text { G4 } & \text { G4 } & \text { G41 } & \text { B } & \text { G4 } & \text { G4 } & \text { G4 } & \text { G41 } \\ \text { B } & \text { G4 } & \text { G3 } & \text { G } & \text { B2 } & \text { B2 } & \text { B23 } & \text { G4 } & \text { G3 } & \text { G41 } & \text { B } & \text { G3 } & \text { G3 } & \text { G4 } & \text { G4 } \\ \text { G3 } & \text { G3 } & \text { G4 } & \text { G4 } & \text { G41 } & \text { B } & \text { G3 } & \text { G4 } & \text { G4 } & \text { G4 } & \text { G } & \text { B23 } & \text { G } & \text { B23 } & \text { G } \\ \text { B23 } & \text { G3 } & \text { G3 } & \text { G3 } & \text { G4 } & \text { G42 } & \text { B1 } & \text { B2 } & \text { B } & \text { G } & \text { B2 } & \text { B23 } & \text { G3 } & \text { G4 } & \text { G4 }\end{array}$

In contrast with the Riemann zeta function, the type sequence is pretty complicated for $L\left(s, E_{5077}\right)$ but does not violate Rosser's law in this range. There exist many reverses of $3 G \rightarrow B 2$ and $G 4 \rightarrow 1 B$. Furthermore, one can find some reverses coming from (30). For example, in Table 10, G42BG is obtained as

$$
G 4 G 4 G \rightarrow G 41 B G \rightarrow G 42 B G .
$$

This observation shows why counterexamples of Rosser's law cannot be found in the short range. However, if we perform the calculation to a wider range, an arbitrary 
complicated change of type sequences would happen, and might violate Rosser's law. We can actually observe such a change at the breaking point of Rosser's law.

\section{ACKNOWLEDGMENTS}

The authors wish to thank Professor L. Murata for his careful reading of the manuscript, and Professor A. Ivić for pointing out the present record of the validity of the Riemann Hypothesis. They also thank the referee for many valuable comments.

\section{REFERENCES}

[1] J. E. Cremona, Algorithms for Modular Elliptic Curves, Cambridge University Press, 1992. MR 93m:11053

[2] F. Diamond, On deformation rings and Hecke rings, preprint.

[3] H. M. Edwards, Riemann's Zeta Function, Academic Press, 1974. MR 57:5922

[4] S. Fermigier, Zéros des Fonction $L$ de Courbes Elliptiques, Experimental Math., 1 (1992), no.2, 167-173. MR 94b:11126

[5] W.B. Jones and W.J. Thron, Continued fractions, Analytic Theory and Applications, Encyclopedia of Math. and its Appl., 11 Addison-Wesley, 1980. MR 82c:30001

[6] W.B. Jones and W.J. Thron, A Posteriori Bounds for the Truncation Error of Continued fractions, SIAM J. Numer. Anal. 8 (1971), 693-705. MR 45:4602

[7] S. Hitotumatu, J. Yamauchi and T. Uno,Sûchikeisanhou III (Numerical Computing Methods III), Baihukan, 1971 (Japanese).

[8] T. Kano (ed.) Riemann yosou (Riemann Hypothesis), Nihonhyouronsha, 1991 (Japanese).

[9] A. A. Karatsuba, Basic Analytic Number Theory, Springer-Verlag, 1991 (English translation). MR 94a:11001

[10] A. W. Knapp, Elliptic curves, Princeton University Press, 1992. MR 93j:11032

[11] L. Kuipers and H. Niederreiter, Uniform Distribution of Sequences, John Wiley \& Sons,New York, 1974. MR 54:7415

[12] A. F. Lavrik, Approximate functional equation for Dirichlet Functions, Izv. Akad. Nauk SSSR 32 (1968), 134-185. MR 36:6361

[13] J.van de Lune, H.J.J.te Riele and D.T.Winter, On the zeros of the Riemann zeta function on the critical strip IV, Math. Comp., 46 (1986), 667-681. MR 87e:11102

[14] Ju. I. Manin, Cyclotomic fields and modular curves, Russian Math. Surveys 26 (1971), 7-71. MR 53:5480

[15] — Parabolic points and zeta-functions of modular curves, Izv. Akad. Nauk SSSR Ser. Mat. 36 (1972), 19-66. MR 47:3396

[16] A.M. Odlyzko, The $10^{20}$-th Zeros of the Riemann Zeta Function and 70 Million of its Neighbors, preprint

[17] A. P. Ogg, A remark on the Sato-Tate conjecture, Invent. Math. 9 (1970), 198-200. MR 41:3481

[18] F. Shahidi, Symmetric power L-functions for GL(2), Elliptic Curves and Related Topics, ed. by H. Kisilevsky and R. Murty, CRM Proc. and Lecture Notes vol 4 (1994), 159-182. MR 95c: 11066

[19] R. Taylor and A. Wiles, Ring-theoretic properties of certain Hecke algebras, Ann. of Math. 114 (1995), 553-572. MR 96d:11072

[20] E.C.Titchmarsh, The Theory of the Riemann Zeta Function (revised by D.R.Heath-Brown) 2nd edition, Oxford University Press, 1986. MR 88c:11049

[21] R. T. Turganaliev, An approximate functional equation and moments of the Dirichlet series generated by the Ramanujan function, Izv. Akad. Nauk Repub. Kazakhstan Ser. Fiz.-Mat. (1992), 49-55 (Russian). MR 94m:11061

[22] H.S. Wall, Analytic theory of continued fractions, Chelsea Publ., 1948. MR 10:32d

[23] A. Wiles, Modular elliptic curves and Fermat's Last Theorem, Ann. of Math. 114 (1995), 443-551. MR 96d:11071

[24] H. Yoshida, On calculations of zeros L-functions related with Ramanujan's discriminant function on the critical line, J. Ramanujan Math. Soc. 3(1) (1988), 87-95. MR 90b:11044 
[25] - On calculations of zeros of various L-functions, Symposium on automorphic forms at Kinosaki (1993), 47-72.

Department of Mathematics, Faculty of Science, Nitgata University, Ikarashi 2-8050, NiIGATA 950-2181, JAPAN

E-mail address: akiyama@math.sc.niigata-u.ac.jp

Graduate School of Mathematics, Nagoya University, Chikusa-Ku, Nagoya 464-8602, JAPAN

E-mail address: tanigawa@math.nagoya-u.ac.jp 